\title{
Synthesis, Electronic Properties and Electrochemiluminescence of Donor-Substituted Phenylethynylanthronitriles
}

\author{
Arumugasamy Elangovan, ${ }^{\dagger}$ Kuo-Ming Kao, ${ }^{\dagger}$ Shu-Wen Yang, ${ }^{\dagger}$ Yu-Ling Chen, ${ }^{\dagger}$ Tong- \\ Ing $\mathrm{Ho}^{* \dagger}$ and Yulong Oliver $\mathrm{Su}^{* *}$ \\ ${ }^{\dagger}$ Department of Chemistry, National Taiwan University, Taipei-106, Taiwan and \\ ${ }^{\ddagger}$ Department of Chemistry, National Ji-Nan University, Taichung, Taiwan
}

\section{Supporting Information}

Contents

Page No.

Synthesis and Characterization data for 1-6

S2

UV-Visible absorption and emission spectra for 1-6 in $\mathrm{CH}_{2} \mathrm{Cl}_{2}$

S4

ECL Spectra of 1-6

$\mathrm{S} 5-\mathrm{S} 7$

${ }^{1} \mathrm{H}$ NMR spectra of 1-6

$\mathrm{S} 8-\mathrm{S} 13$

${ }^{13} \mathrm{C}$ NMR spectra of $\mathbf{1 - 6}$

S14 - S 19

Cartesian coordinates for neutral molecules 1-6 (DFT/B3LYP/6-31G*)

$\mathrm{S} 20-\mathrm{S} 25$

Cartesian coordinates for radical ions of molecules 1 and $\mathbf{6}$ (DFT)

S26 - S29

VU-vis absorption spectra of radical ions of 1-6

$\mathrm{S} 30-\mathrm{S} 35$ 
General procedure for the synthesis of internal alkynes 1-6. 9-Bromo-10cyanoanthracene $(0.5 \mathrm{~m} \mathrm{~mol}$; prepared by bromination of 9-anthronitrile in nitromethane under reflux for $20 \mathrm{~min}$ followed by recrystallization of the precipitate from benzene), palladium catalyst (2 mol \%), $\mathrm{CuI}(2 \mathrm{~mol} \%)$, triphenylphosphine $(10 \mathrm{~mol} \%)$ and a magnetic stirring bar were placed in a two-neck round bottom flask fitted with a condenser. The whole set up was degassed and back-filled with gaseous mixture of argon and hydrogen. To the reaction flask was added previously degassed $5 \mathrm{~mL}$ THF and TEA $(6 \mathrm{mmol})$ using syringes. The terminal acetylene was dissolved in $5 \mathrm{~mL}$ THF and added to the reaction mixture at about $80{ }^{\circ} \mathrm{C}$. The reaction mixture was stirred at reflux for $24 \mathrm{hrs}$ under the atmosphere of the gas mixture. The solvents were evaporated and the crude product was extracted with ether $(25 \mathrm{~mL}+2 \times 15 \mathrm{~mL})$. The combined organic layers were washed with water followed by brine before drying and evaporating. The residue after evaporation was chromatographed on silica gel using ethyl acetate - hexane mixture (1:9) to separate the product 1-6 and unreacted starting compounds.

Characterization data for the compounds 1-6.

10-Phenylethynyl-anthracene-9-carbonitrile (1): Pale yellow solid; mp: $208-211{ }^{\circ} \mathrm{C} ; \mathrm{R}_{\mathrm{f}}$

(hexane) $0.10 ;{ }^{1} \mathrm{H}-\mathrm{NMR}: \delta$ ppm $8.69(\mathrm{~d}, J 8.8 \mathrm{~Hz}, 2 \mathrm{H}), 8.43(\mathrm{~d}, J 8.4 \mathrm{~Hz}, 2 \mathrm{H}), 7.72$ $(\mathrm{m}, \quad 6 \mathrm{H}), 7.46(\mathrm{t}, J \mathrm{~d} 8.0,3 \mathrm{H}) ;{ }^{13} \mathrm{C}-\mathrm{NMR}: \delta$ 132.20, 131.46, 130.99, 129.02, 128.60, 128.29, $127.14,126.90,125.37,123.69,122.23,116.91,105.59,104.62,85.26 ; \mathrm{HR} \mathrm{MS}\left(\mathrm{m} / \mathrm{z} \mathrm{M}^{+}\right)$: 303.1047 Calculated for $\mathrm{C}_{23} \mathrm{H}_{13} \mathrm{~N}$ : 303.1048 .

10-p-Tolylethynyl-anthracene-9-carbonitrile (2): Yellow solid; mp: 229-231 ${ }^{\circ} \mathrm{C} ; \mathrm{R}_{\mathrm{f}}$ (hexane) $0.11{ }^{1} \mathrm{H}-\mathrm{NMR}: \delta 8.78(\mathrm{~d}, J 12 \mathrm{~Hz}, 2 \mathrm{H}), 8.45(\mathrm{~d}, J 12 \mathrm{~Hz}, 2 \mathrm{H}), 7.70(\mathrm{~m}, 6 \mathrm{H})$, $2.43(\mathrm{~s}, 3 \mathrm{H}) ;{ }^{13} \mathrm{C}-\mathrm{NMR} \quad \delta \quad 139.6,132.4,131.6,131.1,129.3,128.8,127.0,125.5,124.2$, 
119.4, 117.1, 105.4, 105.2, 84.8, 21.8; HR MS (m/z M ): 317.1204 Calculated for $\mathrm{C}_{24} \mathrm{H}_{15} \mathrm{~N}$ : 317.1204.

10-(4-Methoxy-phenylethynyl)-anthracene-9-carbonitrile (3): Bright yellow solid; mp: 190-192 ${ }^{\circ} \mathrm{C} ; \mathrm{R}_{\mathrm{f}}$ (hexane) $0.11 ;{ }^{1} \mathrm{H}-\mathrm{NMR}$ (Varian $400 \mathrm{MHz}$ ): $\delta 8.72$ (d, J 8.4 Hz, 2H), 8.45 $(\mathrm{d}, J 8.4 \mathrm{~Hz}, 2 \mathrm{H}), 7.72(\mathrm{~m}, 6 \mathrm{H}), 7.00(\mathrm{~d}, J 6.8 \mathrm{~Hz}, 2 \mathrm{H}), 3.90(\mathrm{~s}, 3 \mathrm{H}) ;{ }^{13} \mathrm{C}-\mathrm{NMR}: \delta=160.4$, $133.3,132.5,131.1,128.8,127.5,126.9,125.6,124.5,117.2,114.6,114.2,105.3,105.1$, 84.4, 55.5; HR MS (m/z M ${ }^{+}$): 333.1154 Calculated for $\mathrm{C}_{24} \mathrm{H}_{15} \mathrm{NO}: 333.1154$.

10-(4-Dimethylamino-phenylethynyl)-anthracene-9-carbonitrile (4): Red needles; mp: 200-202 ${ }^{\circ} \mathrm{C}$; $\mathrm{R}_{\mathrm{f}}$ (hexane) 0.12; ${ }^{1} \mathrm{H}-\mathrm{NMR}: \delta 8.69(\mathrm{~d}, J 8.6 \mathrm{~Hz}, 2 \mathrm{H}), 8.39(\mathrm{~d}, J 8.2 \mathrm{~Hz}, 2 \mathrm{H})$, $7.67(\mathrm{~m}, 6 \mathrm{H}), \quad 6.73(\mathrm{~d}, J \quad 8.9 \mathrm{~Hz}, 2 \mathrm{H}), 3.05(\mathrm{~s}, 6 \mathrm{H}) ;{ }^{13} \mathrm{C}-\mathrm{NMR}: \delta \quad 150.25,132.82,132.40$, $130.56,128.50,127.47,126.40,125.29,125.18,117.29,111.53,108.73,107.37,104.03$, 84.36, 40.43; HR MS (m/z M ): 346.1478 Calculated for $\mathrm{C}_{25} \mathrm{H}_{18} \mathrm{~N}_{2}: 346.1470$.

10-(4-Diethylamino-phenylethynyl)-anthracene-9-carbonitrile (5): Bright fine red needles; mp: $198-200{ }^{\circ} \mathrm{C}$; $\mathrm{R}_{\mathrm{f}}$ (hexane) $0.15 ;{ }^{1} \mathrm{H}-\mathrm{NMR}: \delta 8.72(\mathrm{~d}, J \quad 8.5 \mathrm{~Hz}, 2 \mathrm{H}), 8.40(\mathrm{~d}, J$ $8.5 \mathrm{~Hz}, 2 \mathrm{H}), 7.67(\mathrm{~m}, 4 \mathrm{H}), 6.70(\mathrm{~d}, J 8.9 \mathrm{~Hz}, 2 \mathrm{H}), 3.43(\mathrm{q}, 4 \mathrm{H}), 1.21(\mathrm{t}, 6 \mathrm{H}) ;{ }^{13} \mathrm{C}-\mathrm{NMR}: \delta$ $148.2,133.4,132.6,130.7,128.7,127.7,126.5,125.5,125.4,117.5,111.1,107.8,103.9$, 84.3, 44.5, 12.7; HR MS (m/z M $\left.{ }^{+}\right): 374.1780$ Calculated for $\mathrm{C}_{27} \mathrm{H}_{22} \mathrm{~N}_{2}: 374.1783$.

10-(4-Piperidin-1-yl-phenylethynyl)-anthracene-9-carbonitrile (6): Red needles; mp: 205-207 ${ }^{\circ} \mathrm{C}$; $\mathrm{R}_{\mathrm{f}}$ (hexane) $0.14 ;{ }^{1} \mathrm{H}-\mathrm{NMR}: \delta 8.71(\mathrm{~d}, J 8.5 \mathrm{~Hz}, 2 \mathrm{H}), 8.42(\mathrm{~d}, J 8.5 \mathrm{~Hz}, 2 \mathrm{H})$, $7.64(\mathrm{~m}, 4 \mathrm{H}), 7.37$ (d, $J 8.9 \mathrm{~Hz}, 2 \mathrm{H}), 6.97(\mathrm{~d}, J 8.9 \mathrm{~Hz}, 2 \mathrm{H}), 3.31$ (t, $J 14.3 \mathrm{~Hz}, 4 \mathrm{H}), 1.65$ $(\mathrm{m}, 6 \mathrm{H}) ;{ }^{13} \mathrm{C}-\mathrm{NMR}: \delta 151.8,133.4,133.1,133.0,130.8,128.9,127.6,127.2,125.5,125.0$, 117.4, 114.8, 106.8, 104.3, 84.4, 49.3, 25.5, 24.3; HR MS (m/z M+ $)$ : 386.1783 Calculated for $\mathrm{C}_{28} \mathrm{H}_{22} \mathrm{~N}_{2}: 386.1783$. 


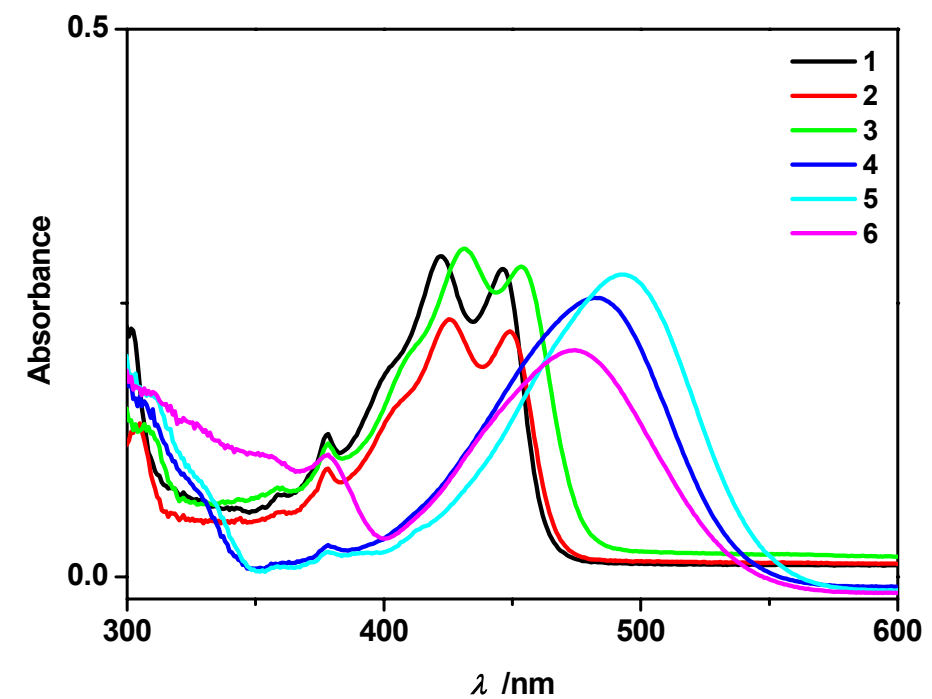

Figure S1. UV-Visible absorption spectra of PEAN 1-6 $\left(1 \times 10^{-5} \mathrm{M} \mathrm{CH}_{2} \mathrm{Cl}_{2}\right)$.

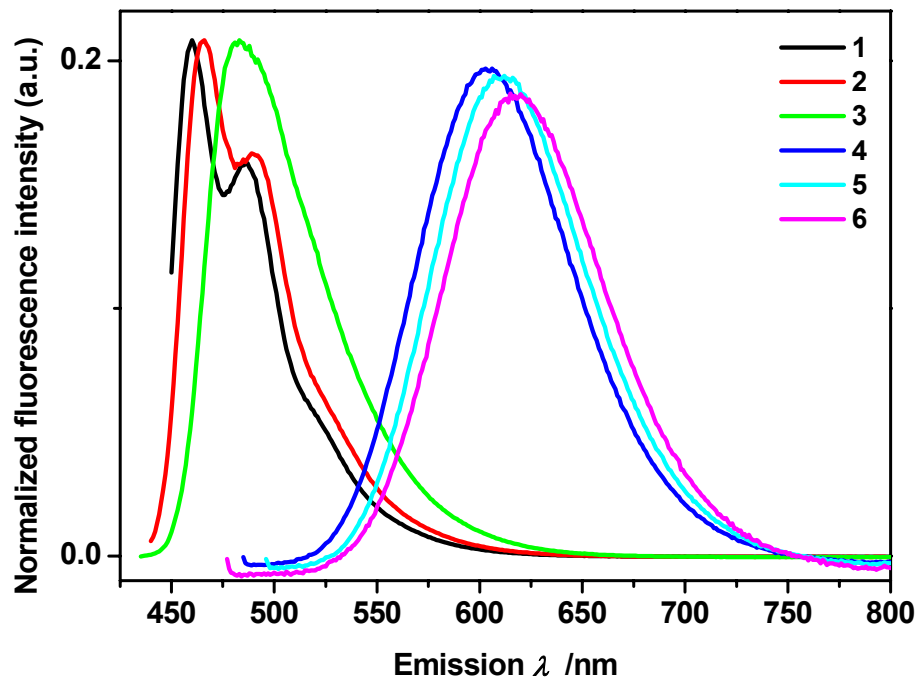

Figure S2. Fluorescence emission spectra of PEAN 1-6 in $\mathrm{CH}_{2} \mathrm{Cl}_{2}\left(1 \times 10^{-5} \mathrm{M}\right.$; 4-6 not normalized). 


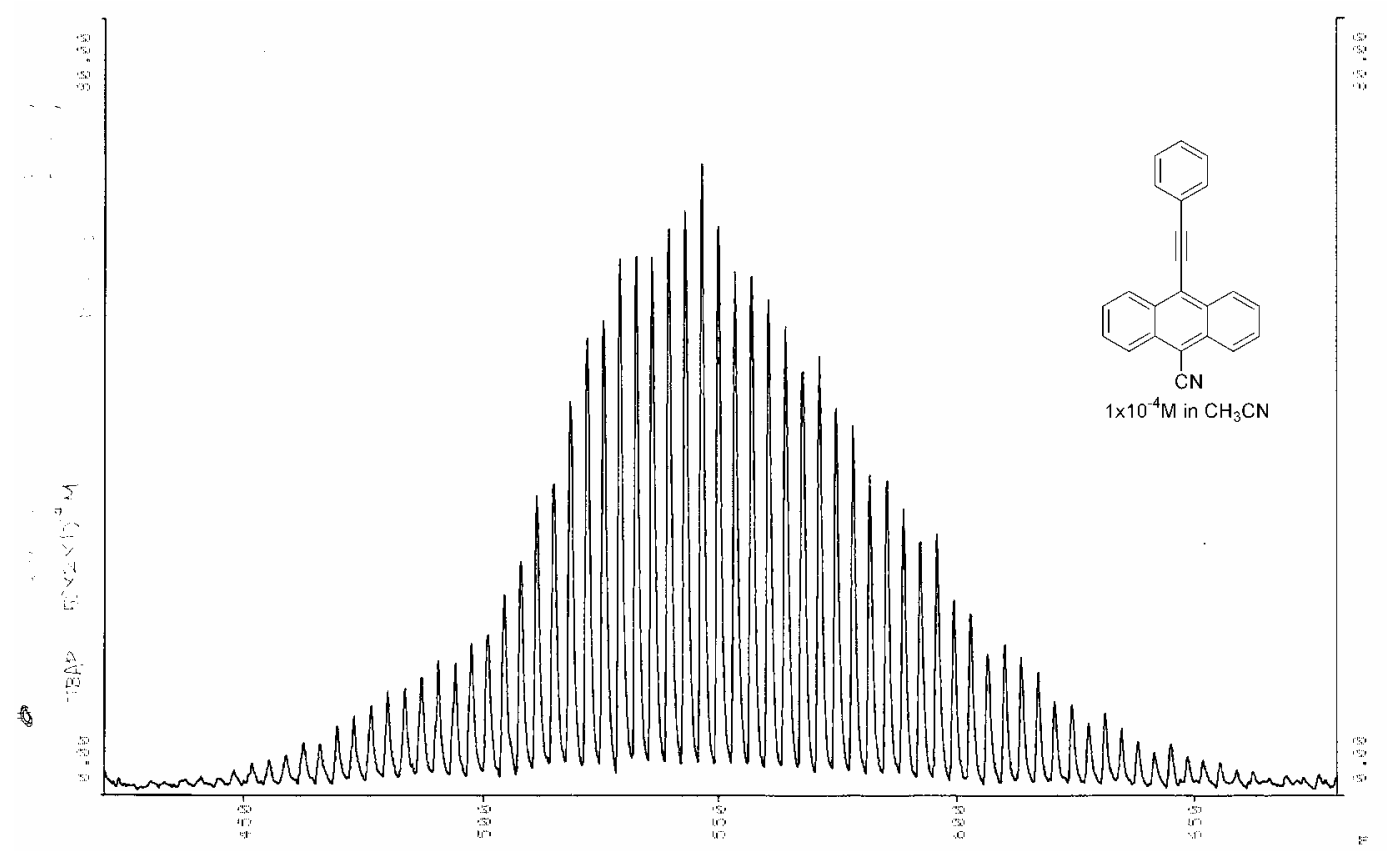

Figure S3-1.

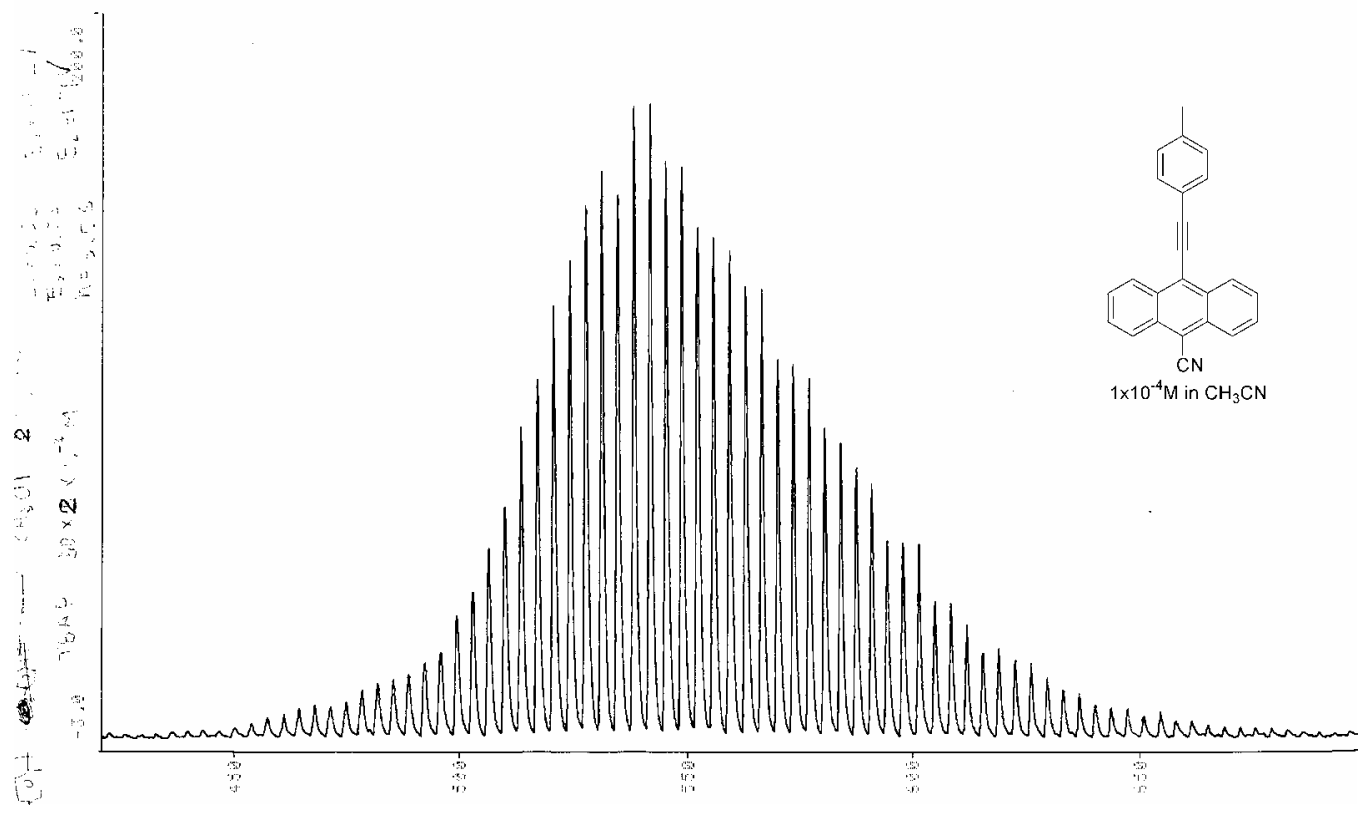

Figure S3-2. 


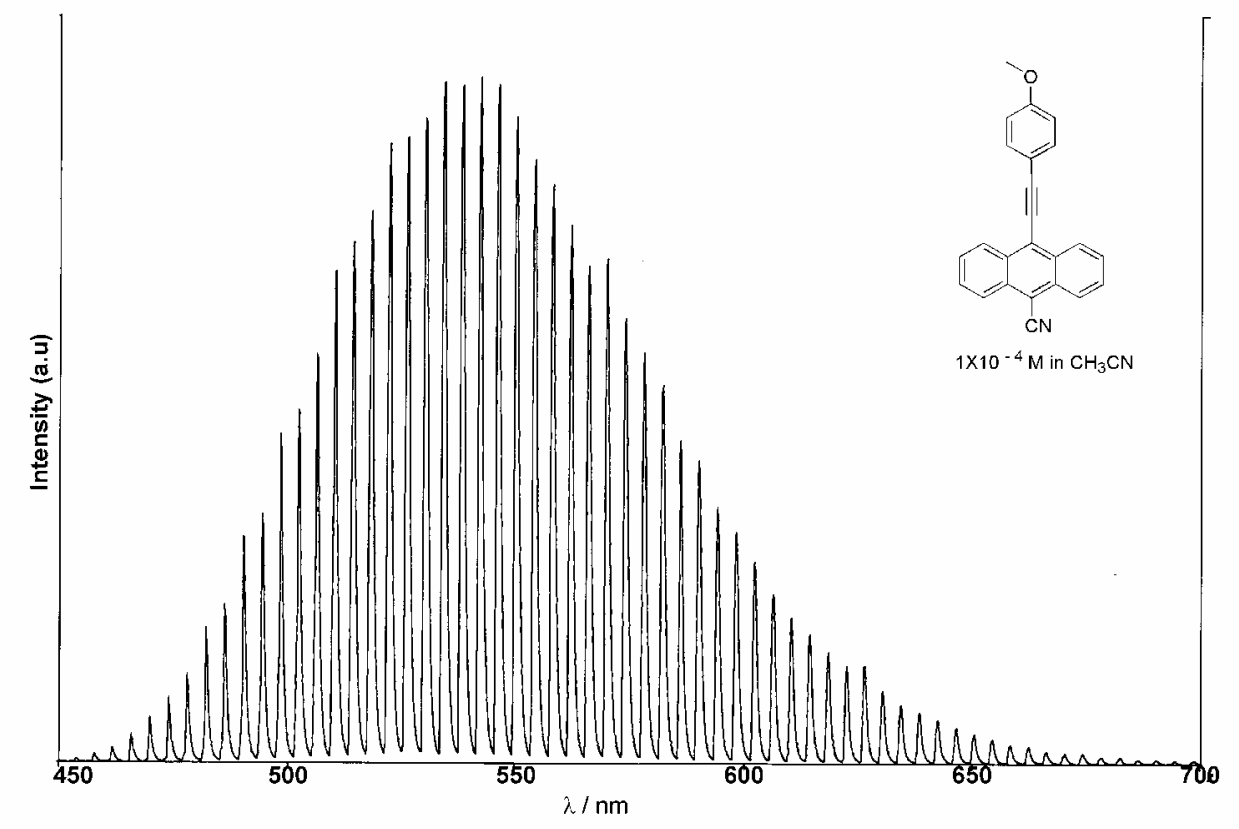

Figure S3-3.

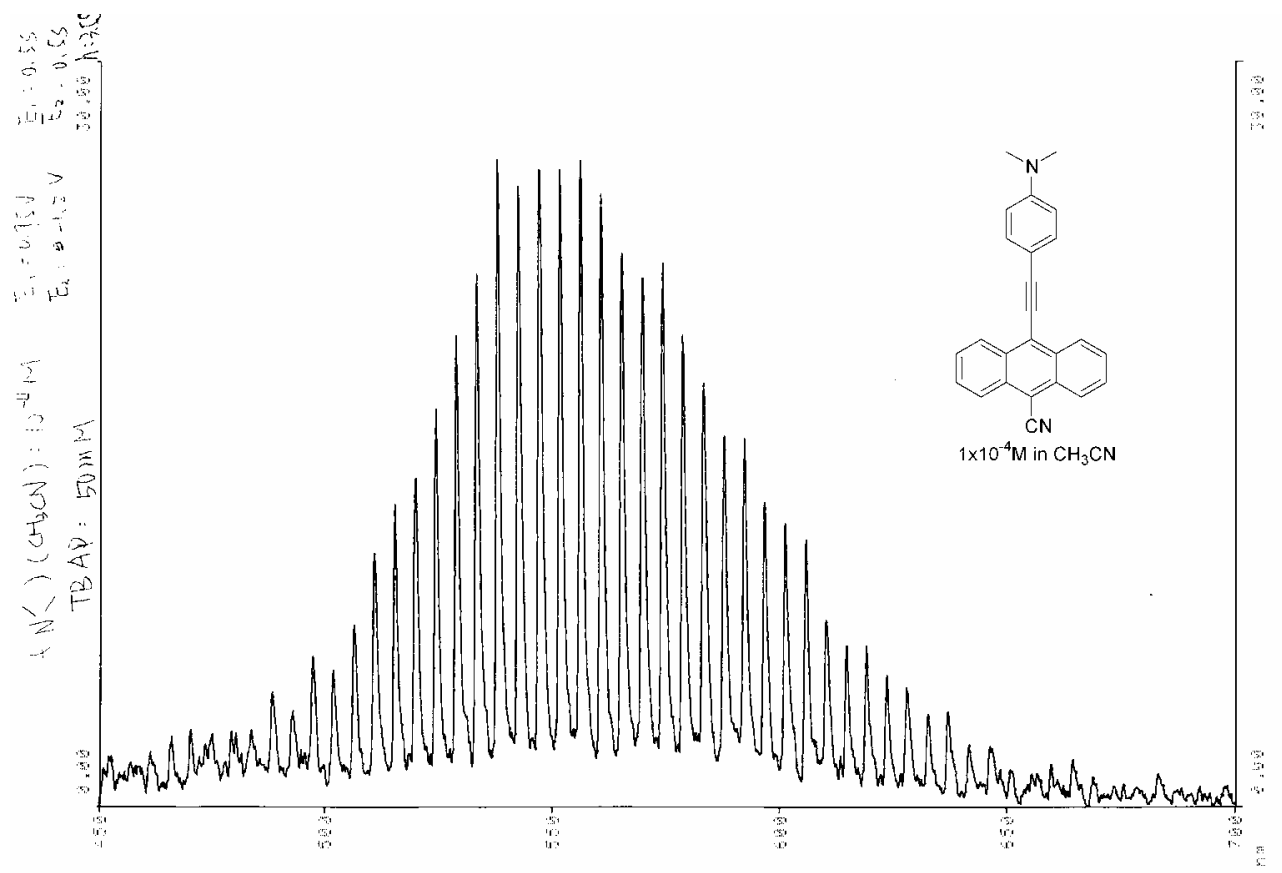

Figure S3-4. 


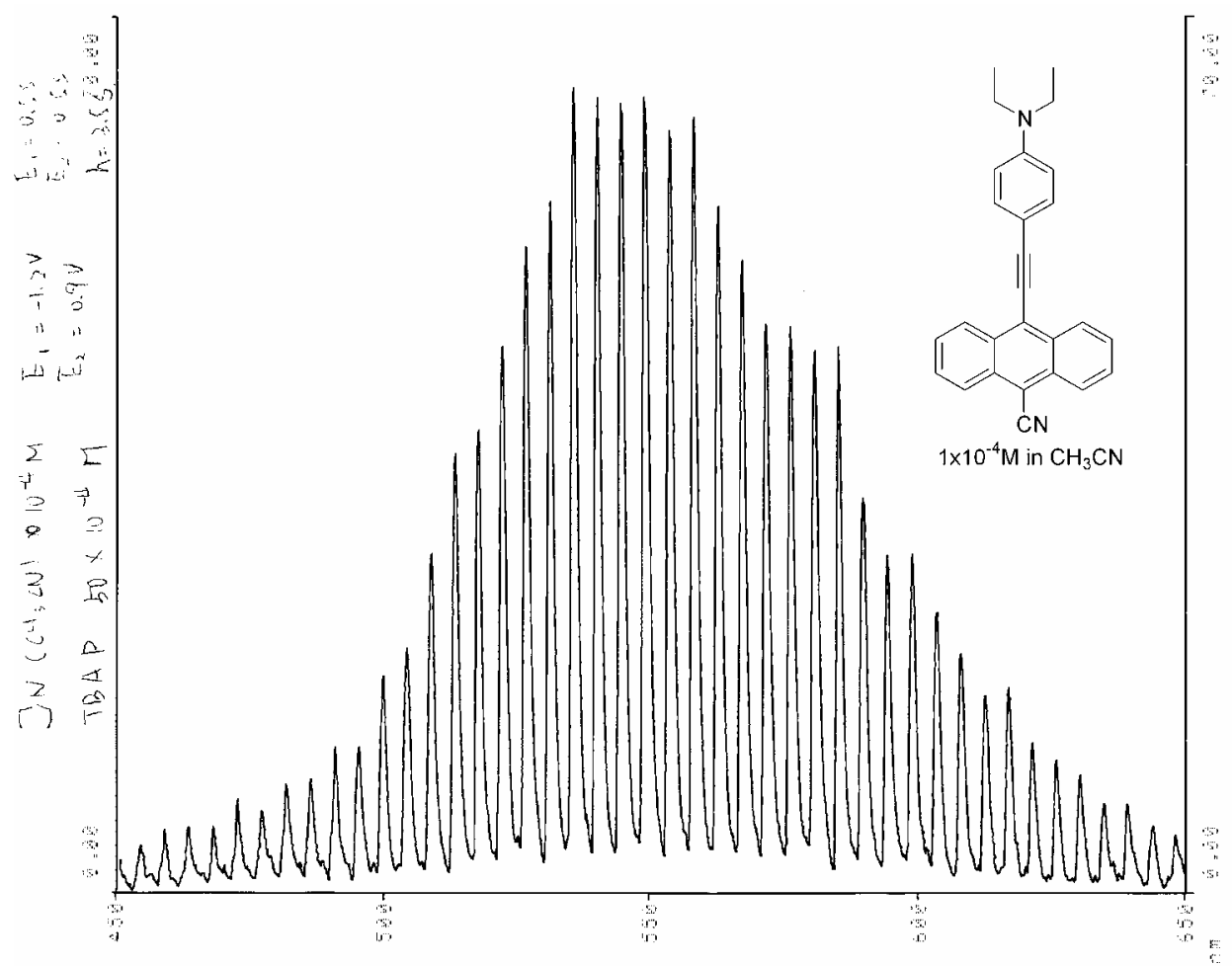

Figure S3-5.

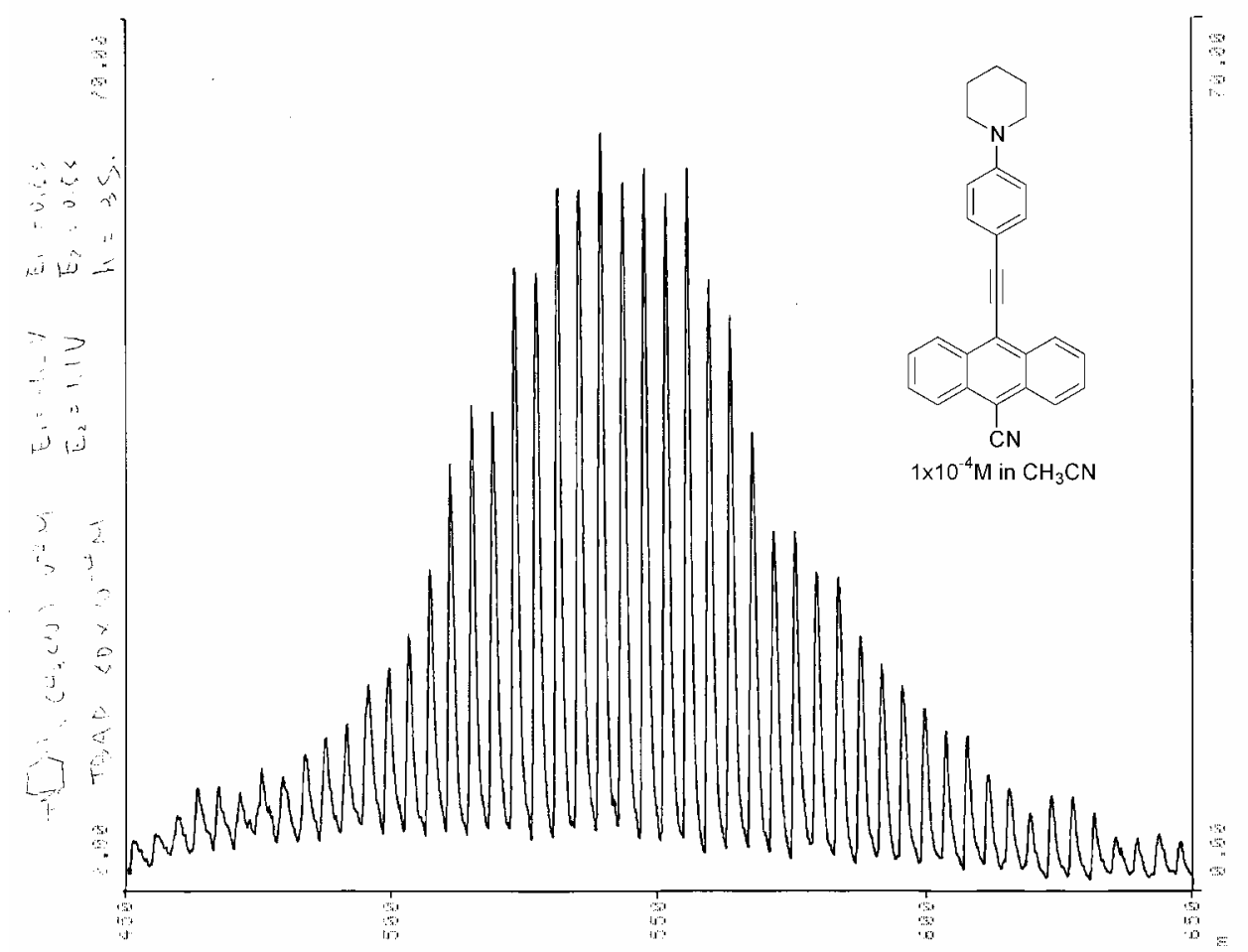

Figure S3-6.

Figure S3. ECL spectra of 1-6 in $\mathrm{CH}_{3} \mathrm{CN}\left(\mathrm{c}=10^{-5} \mathrm{M}\right)$. 


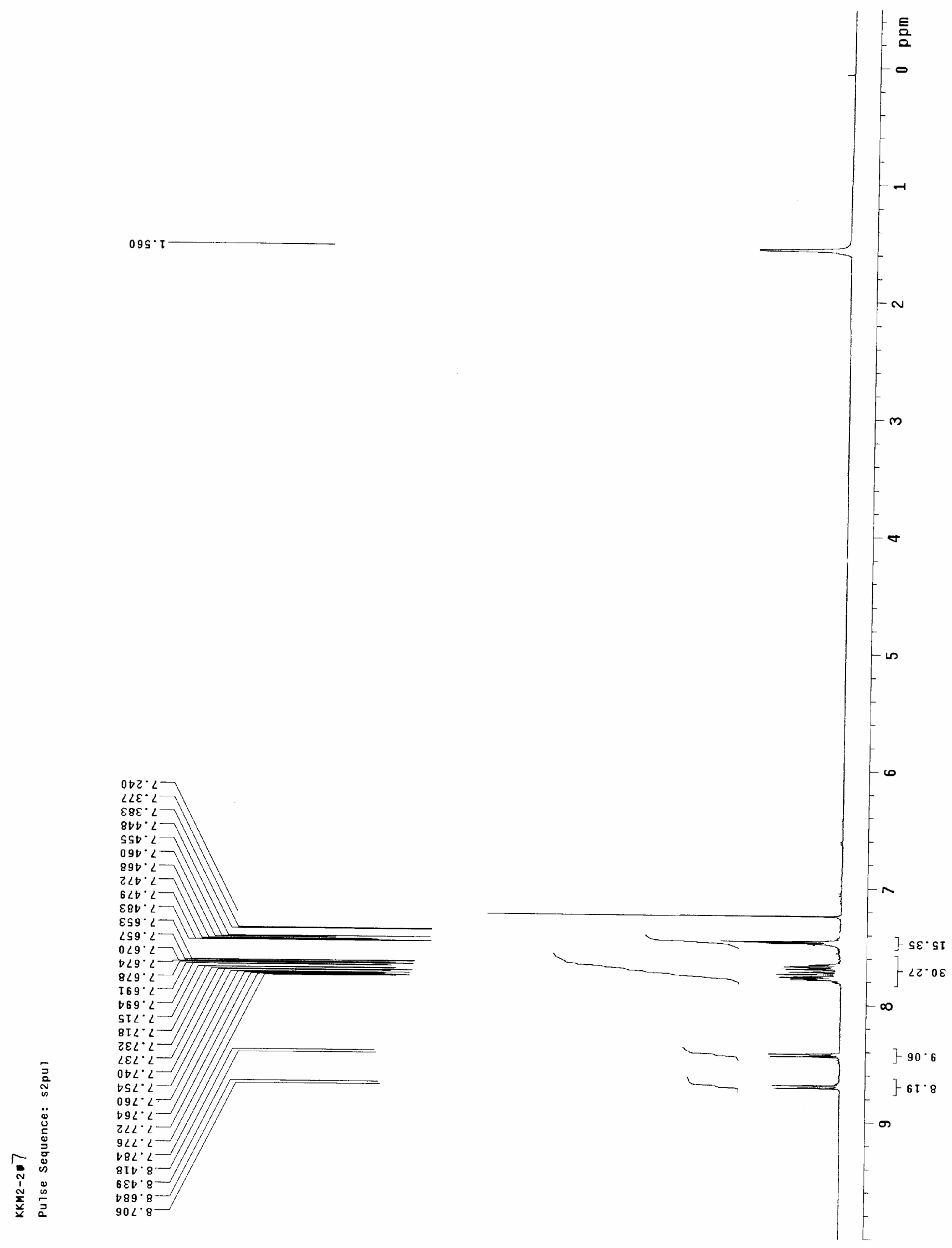

Figure S4-1. ${ }^{1} \mathrm{H}-\mathrm{NMR}$ spectrum of $\mathbf{1 .}$ 


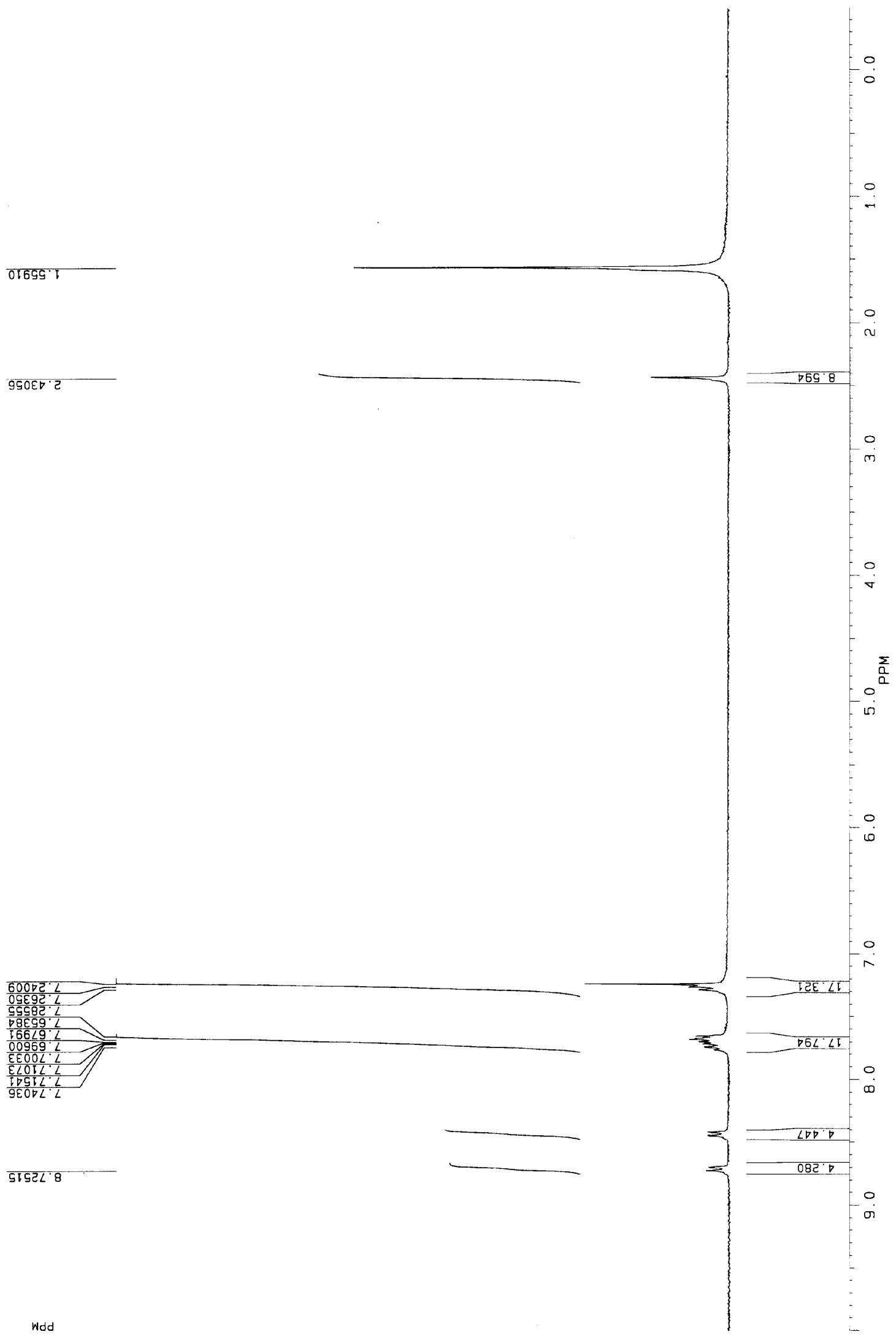

Figure S4-2. ${ }^{1} \mathrm{H}-\mathrm{NMR}$ spectrum of 2. 
$\overline{08 \angle 59 \cdot \tau}$
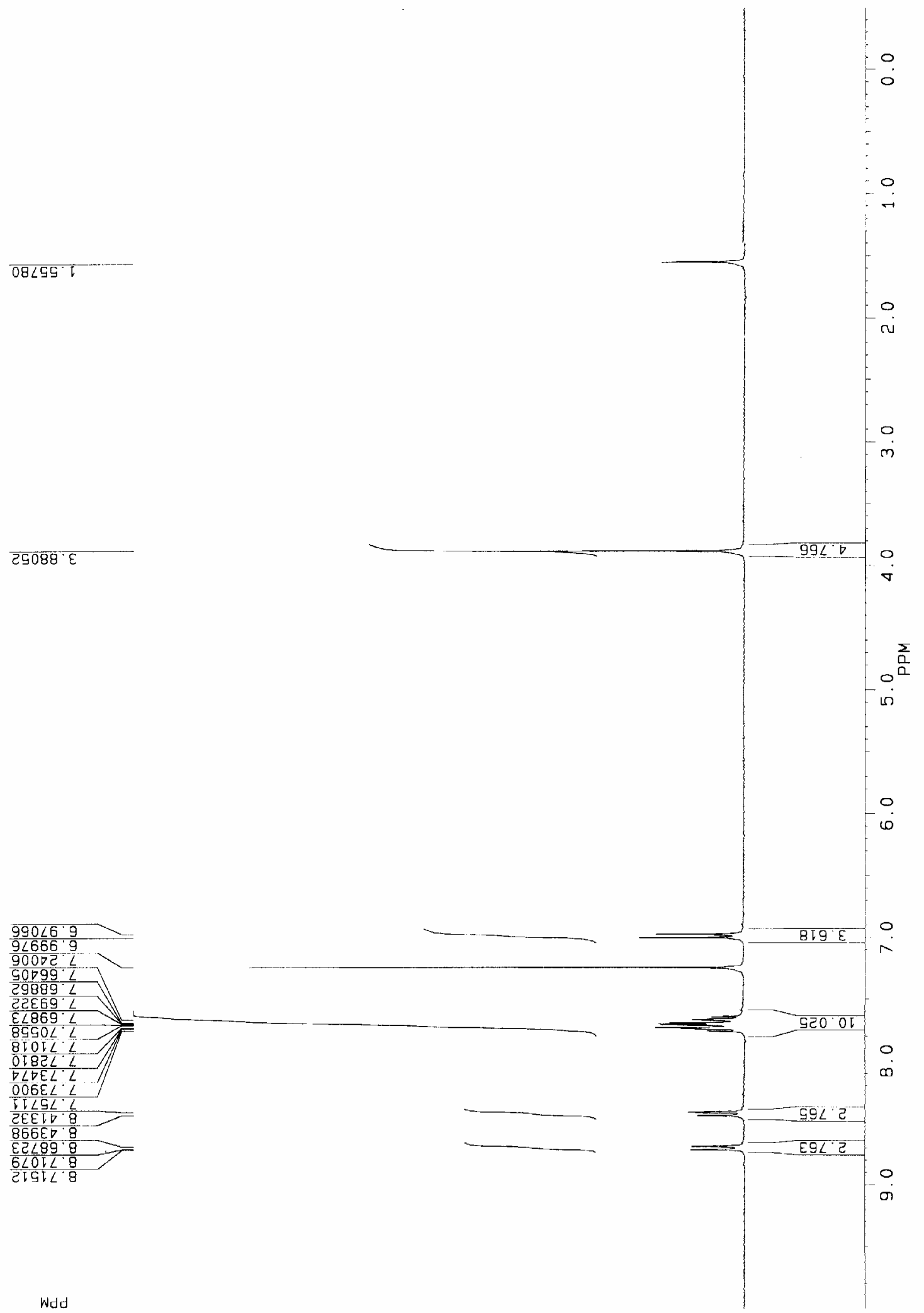

Figure S4-3. ${ }^{1} \mathrm{H}-\mathrm{NMR}$ spectrum of 3 . 


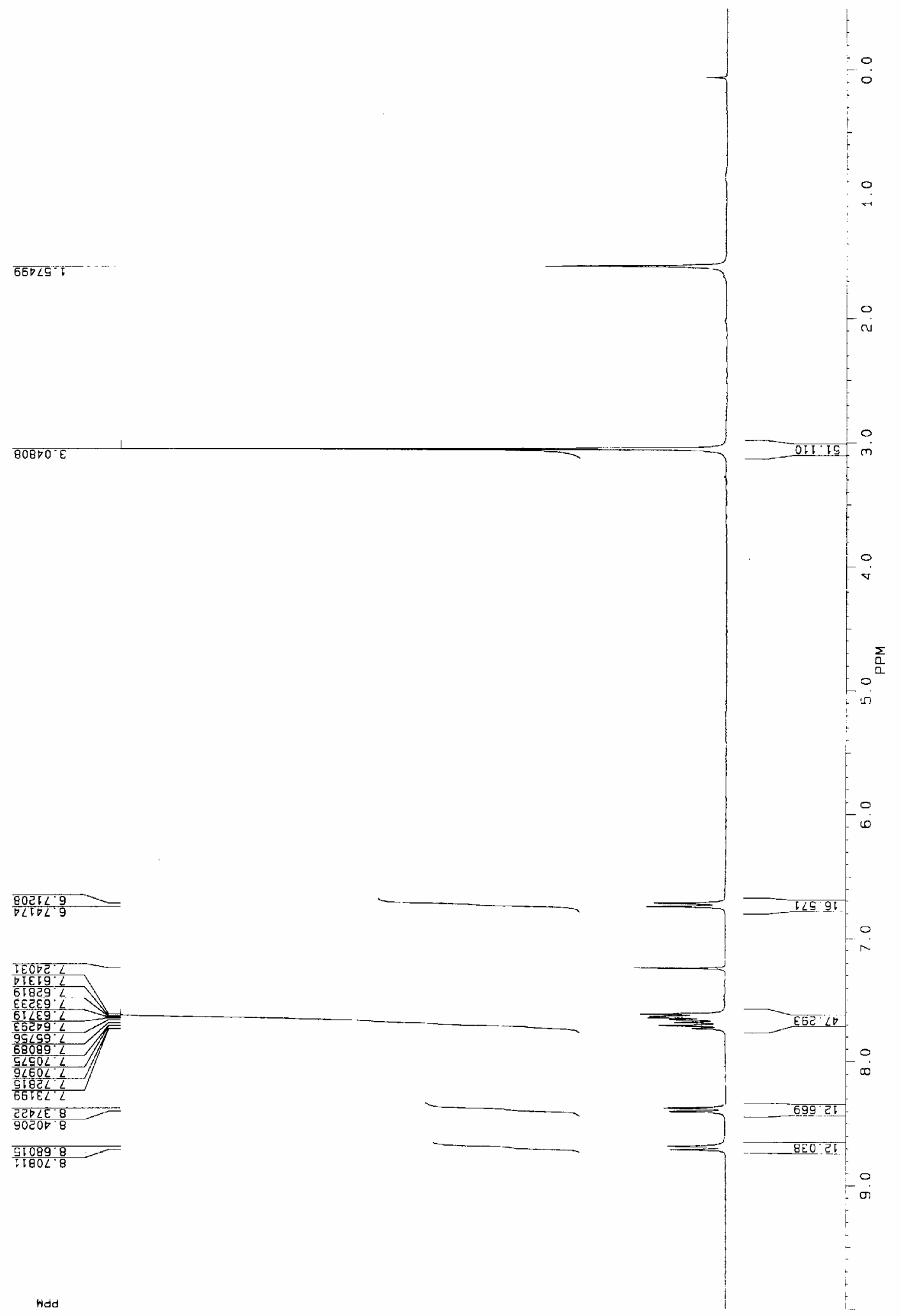

Figure S4-4. ${ }^{1} \mathrm{H}-\mathrm{NMR}$ spectrum of 4. 


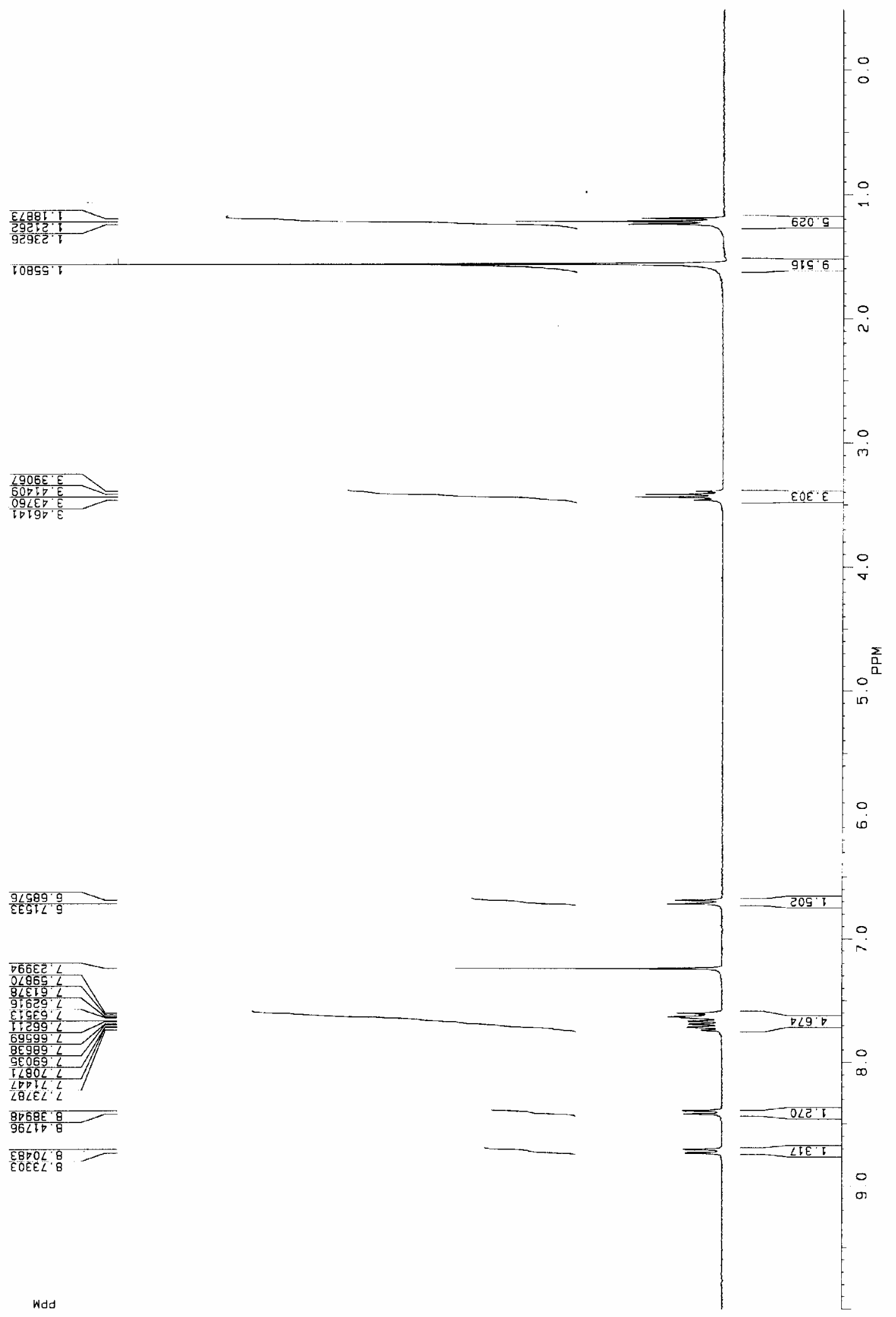

Figure S4-5. ${ }^{1}$ H-NMR spectrum of 5 . 

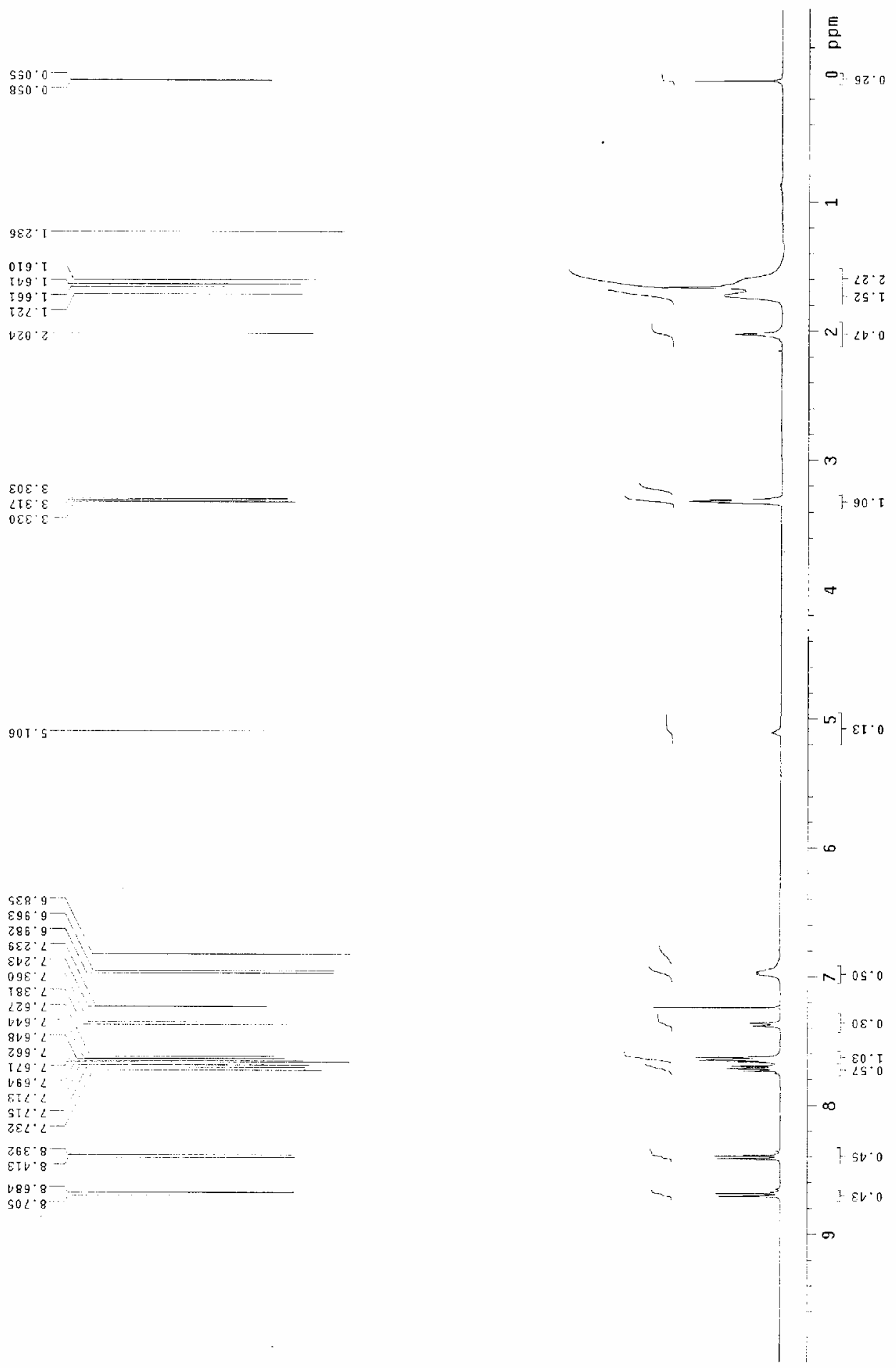

Figure S4-6. ${ }^{1} \mathrm{H}-\mathrm{NMR}$ spectrum of $\mathbf{6}$. 


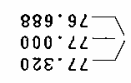

$\varepsilon 9 z_{\leftarrow} \cdot s 8$

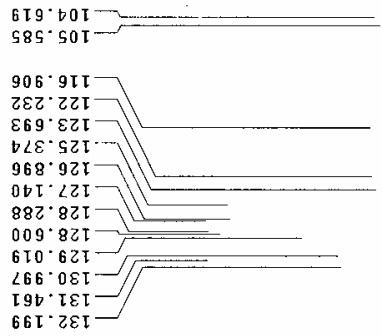

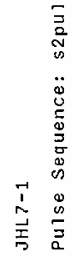

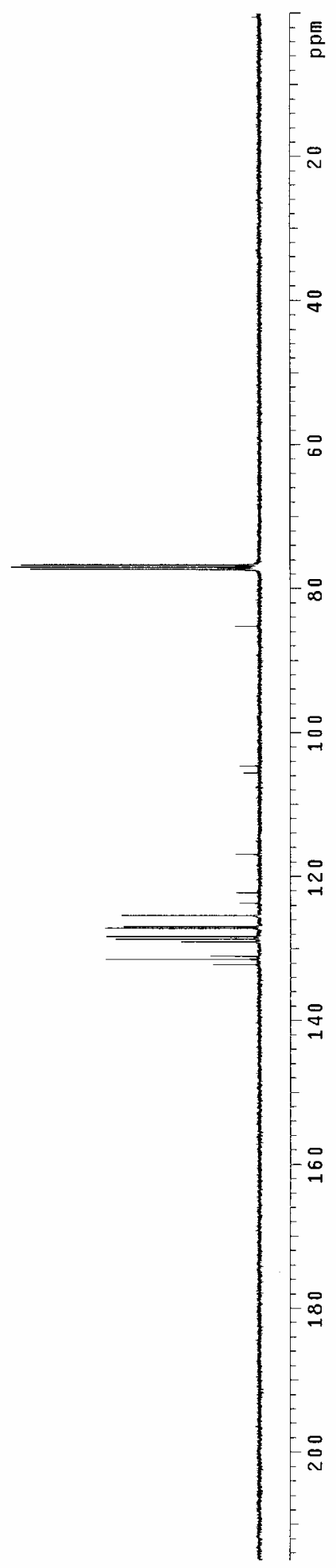

Figure S5-1. ${ }^{13} \mathrm{C}-\mathrm{NMR}$ spectrum of $\mathbf{1}$. 


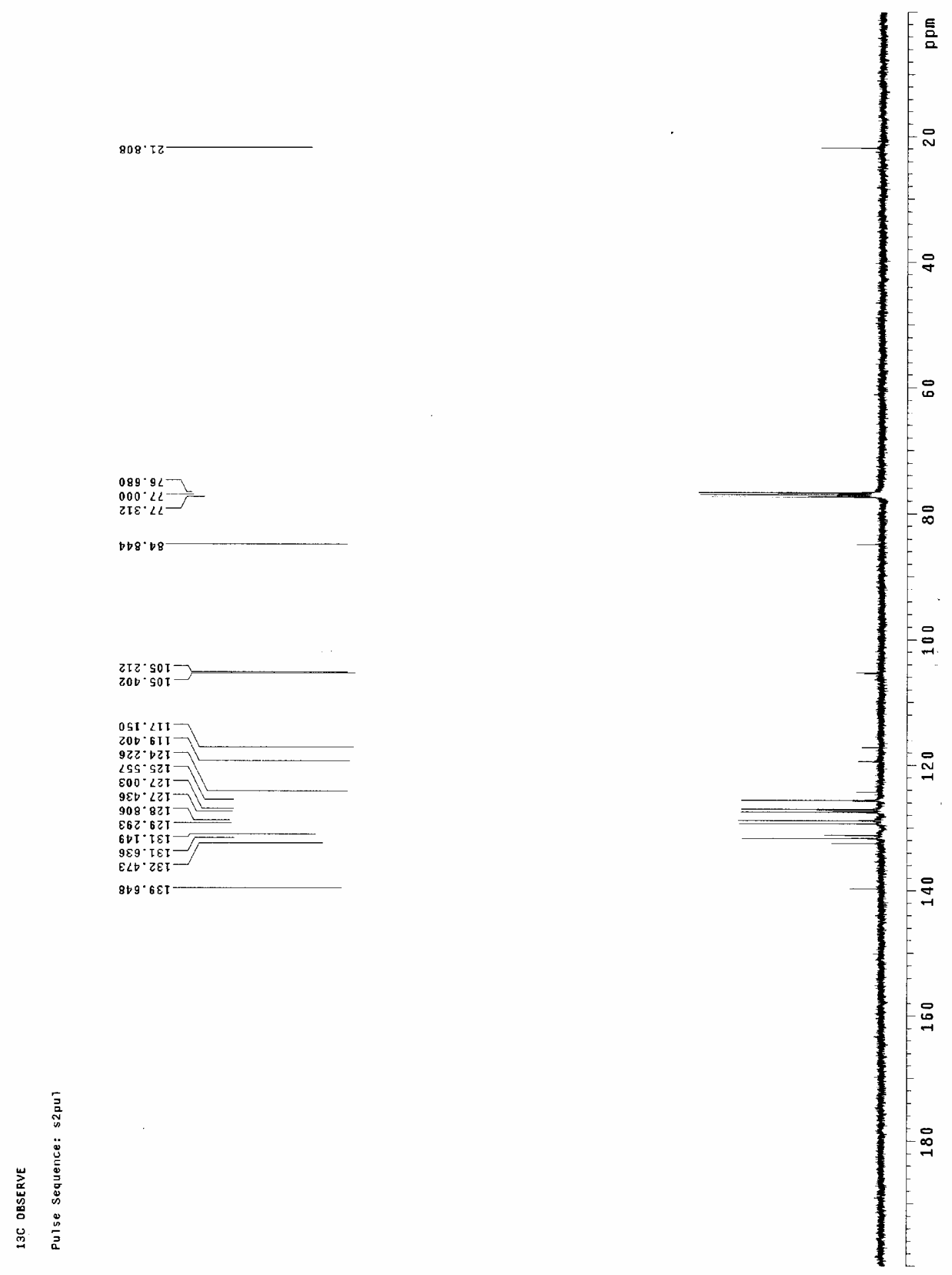

Figure S5-2. ${ }^{13} \mathrm{C}-\mathrm{NMR}$ spectrum of $\mathbf{2}$. 


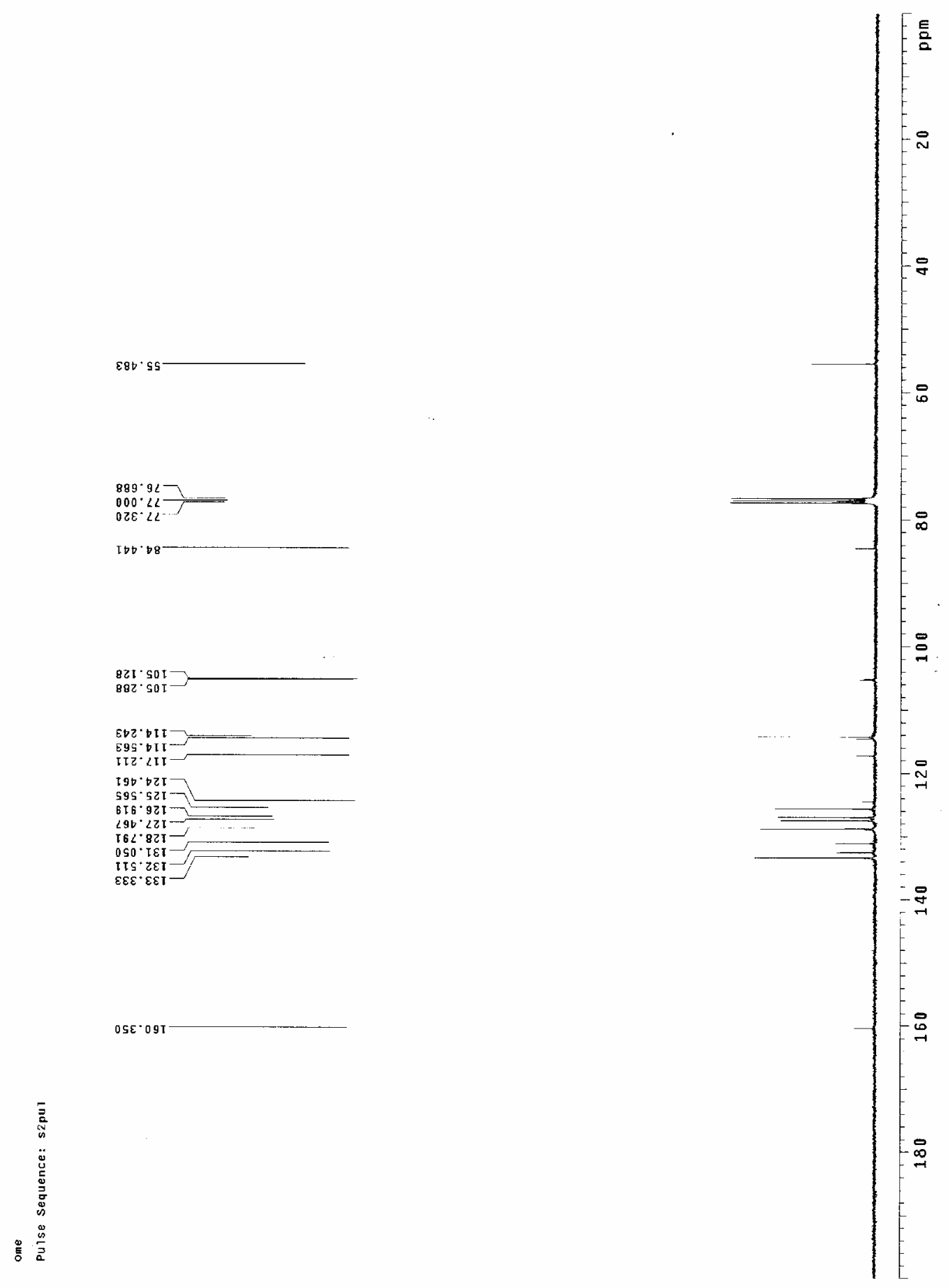

Figure S5-3. ${ }^{13} \mathrm{C}-\mathrm{NMR}$ spectrum of $\mathbf{3}$. 

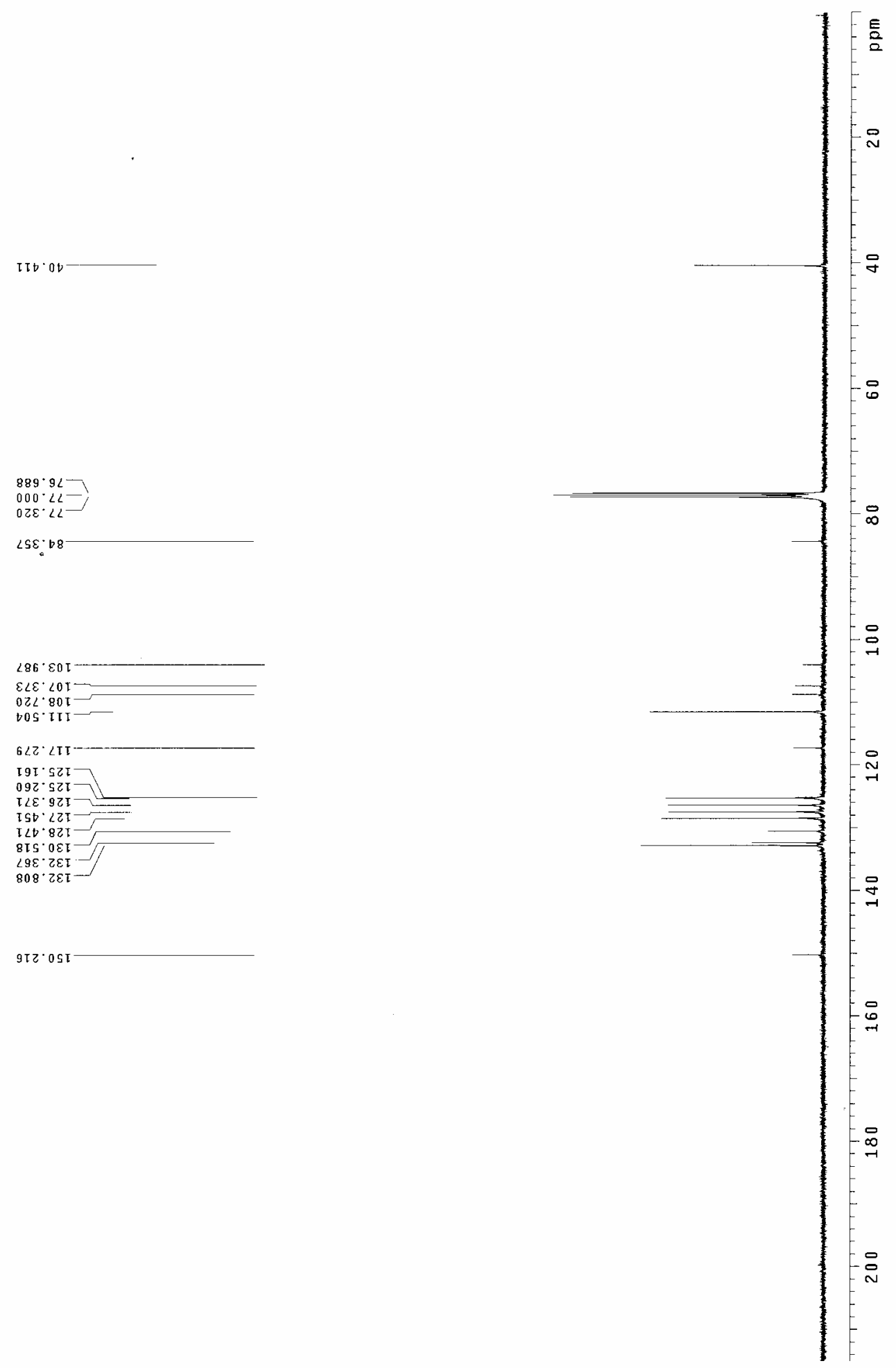

Figure S5-4. ${ }^{13} \mathrm{C}-\mathrm{NMR}$ spectrum of $\mathbf{4}$. 

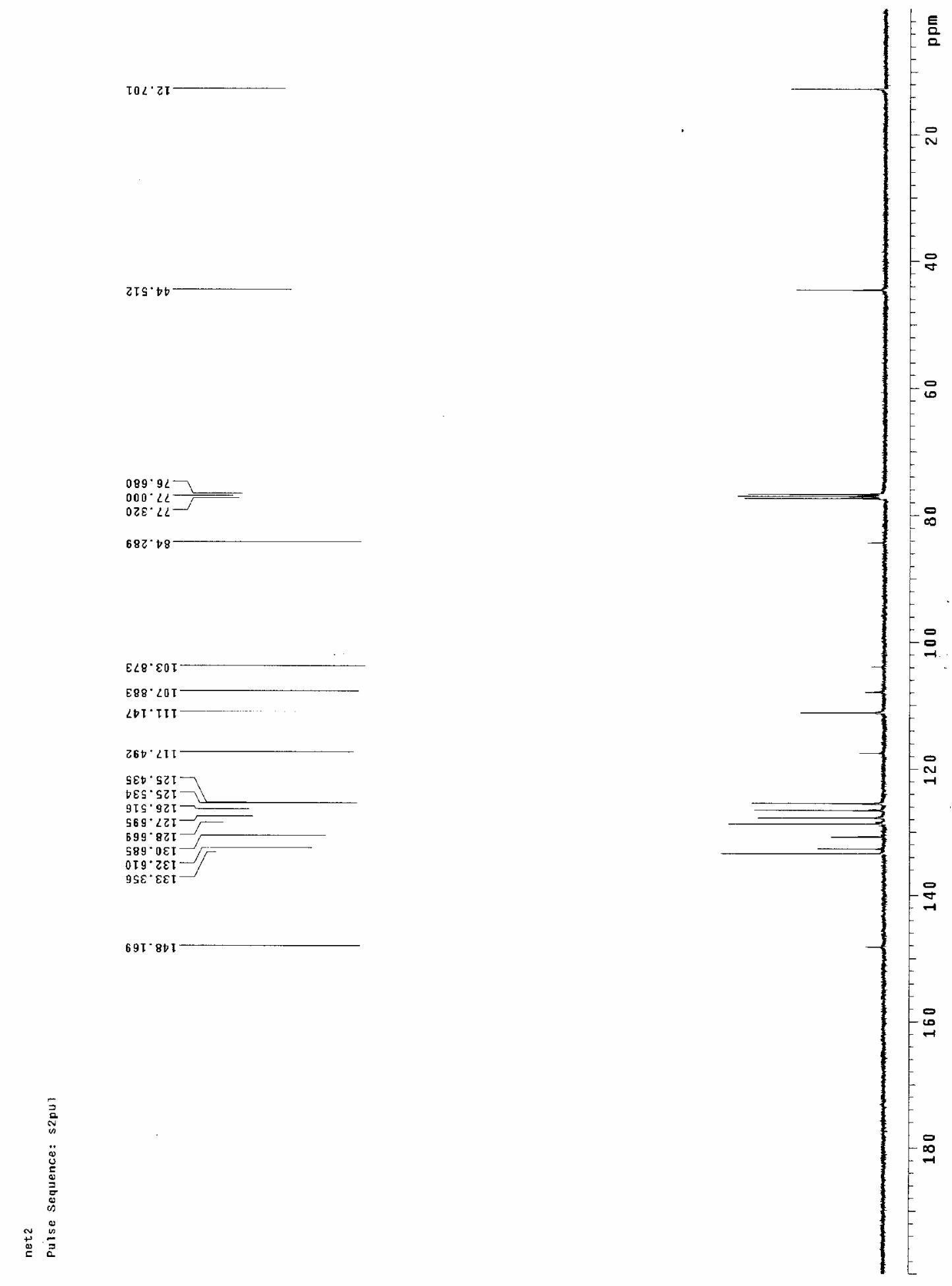

Figure S5-5. ${ }^{13} \mathrm{C}-\mathrm{NMR}$ spectrum of 5. 

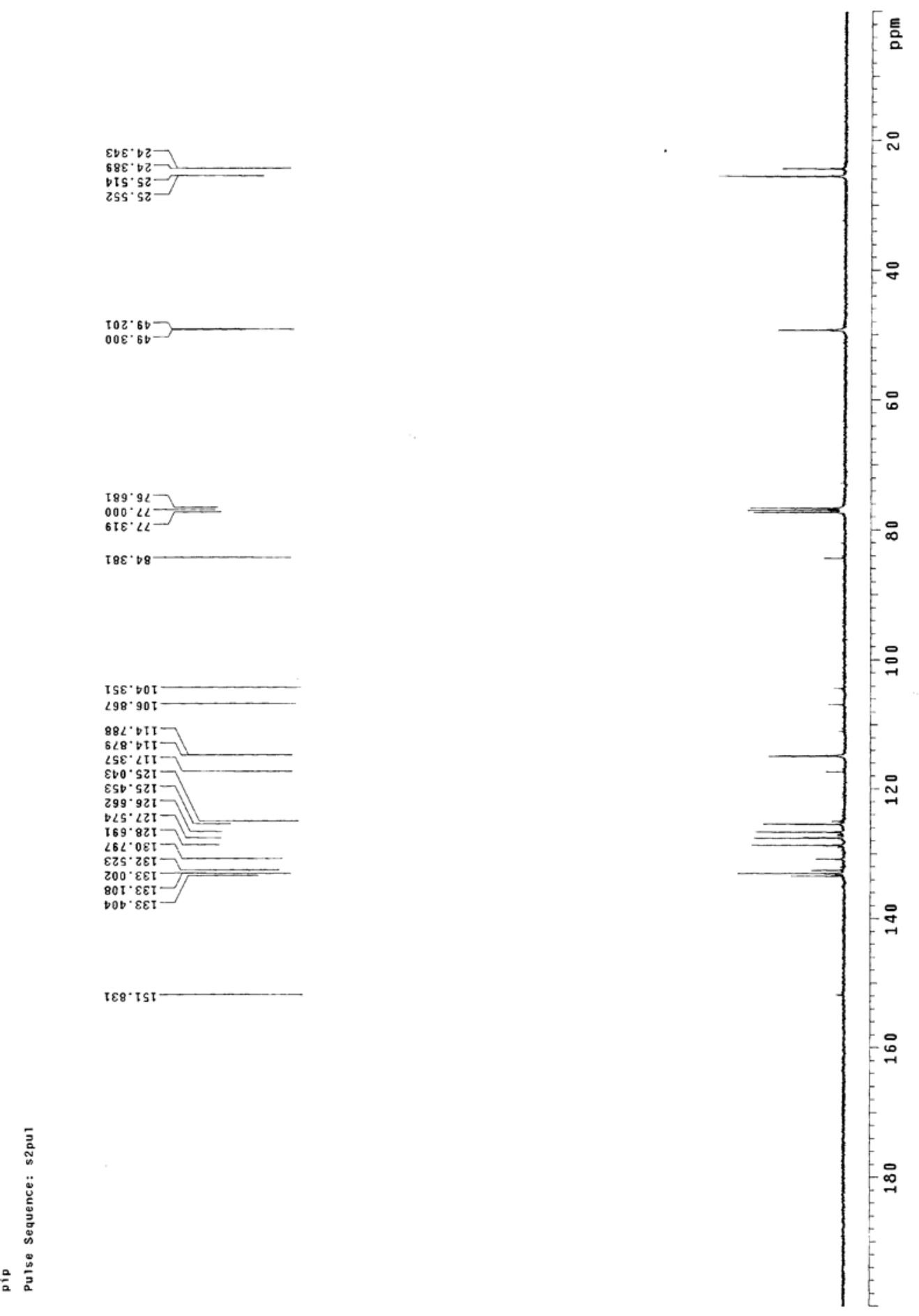

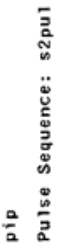

Figure S5-6. ${ }^{13} \mathrm{C}-\mathrm{NMR}$ spectrum of $\mathbf{6}$. 
PEAN 1 (neutral, DFT/ B3LYP/6-31G*) Energy: -938.99 au; Imaginary frequency: none

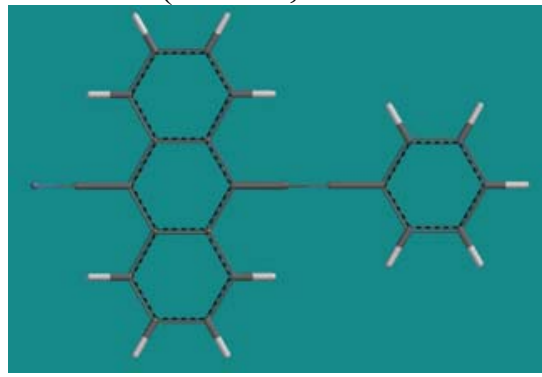

\begin{tabular}{|c|c|c|c|c|c|}
\hline & & & an Coordin & (Angstroms) & \\
\hline & & Atom & $\mathrm{x}$ & Y & Z \\
\hline 1 & $\mathrm{H}$ & $\mathrm{H} 1$ & -4.6126690 & 0.0000000 & -3.3722513 \\
\hline 2 & $\mathrm{C}$ & $\mathrm{C} 1$ & -3.6699533 & 0.0000000 & -2.8325430 \\
\hline 31 & $\mathrm{H}$ & H6 & -2.4843945 & 0.0000000 & -4.6096261 \\
\hline 4 & $\mathrm{C}$ & $\mathrm{C} 6$ & -2.4867199 & 0.0000000 & -3.5241714 \\
\hline 5 & $\mathrm{C}$ & C3 & -2.4896717 & 0.0000000 & -0.7186847 \\
\hline 6 & $\mathrm{C}$ & C5 & -1.2361342 & 0.0000000 & -2.8363032 \\
\hline 7 & $\mathrm{C}$ & C2 & -3.6721603 & 0.0000000 & -1.4119997 \\
\hline 8 & $\mathrm{C}$ & C4 & -1.2361180 & 0.0000000 & -1.3985802 \\
\hline 9 & $\mathrm{C}$ & $\mathrm{C} 10$ & 0.0000000 & 0.0000000 & -3.5273425 \\
\hline 101 & $\mathrm{H}$ & $\mathrm{H} 2$ & -4.6166961 & 0.0000000 & -0.8755397 \\
\hline 111 & $\mathrm{H}$ & H3 & -2.4862548 & 0.0000000 & 0.3658963 \\
\hline 12 & $\mathrm{C}$ & C9 & 1.2361342 & 0.0000000 & -2.8363032 \\
\hline 13 & $\mathrm{C}$ & C14 & 2.4867199 & 0.0000000 & -3.5241714 \\
\hline 14 & $\mathrm{C}$ & $\mathrm{C} 8$ & 1.2361180 & 0.0000000 & -1.3985802 \\
\hline 15 & $\mathrm{H}$ & $\mathrm{H} 11$ & 2.4862548 & 0.0000000 & 0.3658963 \\
\hline 16 & $\mathrm{C}$ & C7 & 0.0000000 & 0.0000000 & -0.6925762 \\
\hline 17 & $\mathrm{C}$ & C13 & 3.6699533 & 0.0000000 & -2.8325430 \\
\hline 18 & $\mathrm{H}$ & H14 & 2.4843945 & 0.0000000 & -4.6096261 \\
\hline 19 & $\mathrm{H}$ & $\mathrm{H} 13$ & 4.6126690 & 0.0000000 & -3.3722513 \\
\hline 20 & $\mathrm{C}$ & $\mathrm{C} 12$ & 3.6721603 & 0.0000000 & -1.4119997 \\
\hline 21 & $\mathrm{H}$ & $\mathrm{H} 12$ & 4.6166961 & 0.0000000 & -0.8755397 \\
\hline 22 & $\mathrm{C}$ & $\mathrm{C} 11$ & 2.4896717 & 0.0000000 & -0.7186847 \\
\hline 23 & $\mathrm{C}$ & C15 & 0.0000000 & 0.0000000 & 0.7260655 \\
\hline 24 & $\mathrm{C}$ & C16 & 0.0000000 & 0.0000000 & 1.9448817 \\
\hline 25 & $\mathrm{C}$ & C17 & 0.0000000 & 0.0000000 & 3.3686487 \\
\hline 26 & $\mathrm{C}$ & $\mathrm{C} 18$ & 0.0000000 & 0.0000000 & 6.1777692 \\
\hline 27 & $\mathrm{C}$ & C19 & -1.2139413 & 0.0000000 & 4.0859107 \\
\hline 28 & $\mathrm{C}$ & $\mathrm{C} 20$ & 1.2139413 & 0.0000000 & 4.0859107 \\
\hline 29 & $\mathrm{C}$ & C21 & 1.2089173 & 0.0000000 & 5.4773914 \\
\hline 30 & $\mathrm{C}$ & C22 & -1.2089173 & 0.0000000 & 5.4773914 \\
\hline 31 & $\mathrm{H}$ & H4 & -2.1526111 & 0.0000000 & 3.5404284 \\
\hline 32 & $\mathrm{H}$ & H5 & 2.1526111 & 0.0000000 & 3.5404284 \\
\hline 331 & $\mathrm{H}$ & H7 & 2.1512732 & 0.0000000 & 6.0180989 \\
\hline 341 & $\mathrm{H}$ & H8 & -2.1512732 & 0.0000000 & 6.0180989 \\
\hline 351 & $\mathrm{H}$ & H9 & 0.0000000 & 0.0000000 & 7.2642518 \\
\hline 36 & $\mathrm{C}$ & $\mathrm{C} 23$ & 0.0000000 & 0.0000000 & -4.9562716 \\
\hline 371 & $\mathrm{~N}$ & N1 & 0.0000000 & 0.0000000 & -6.1214792 \\
\hline
\end{tabular}


PEAN 2 (neutral, DFT/ B3LYP/6-31G*) Energy: -978.31 au; Imaginary frequency: none

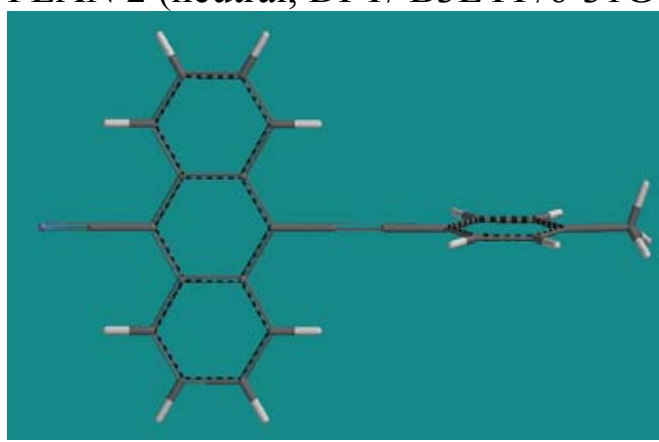

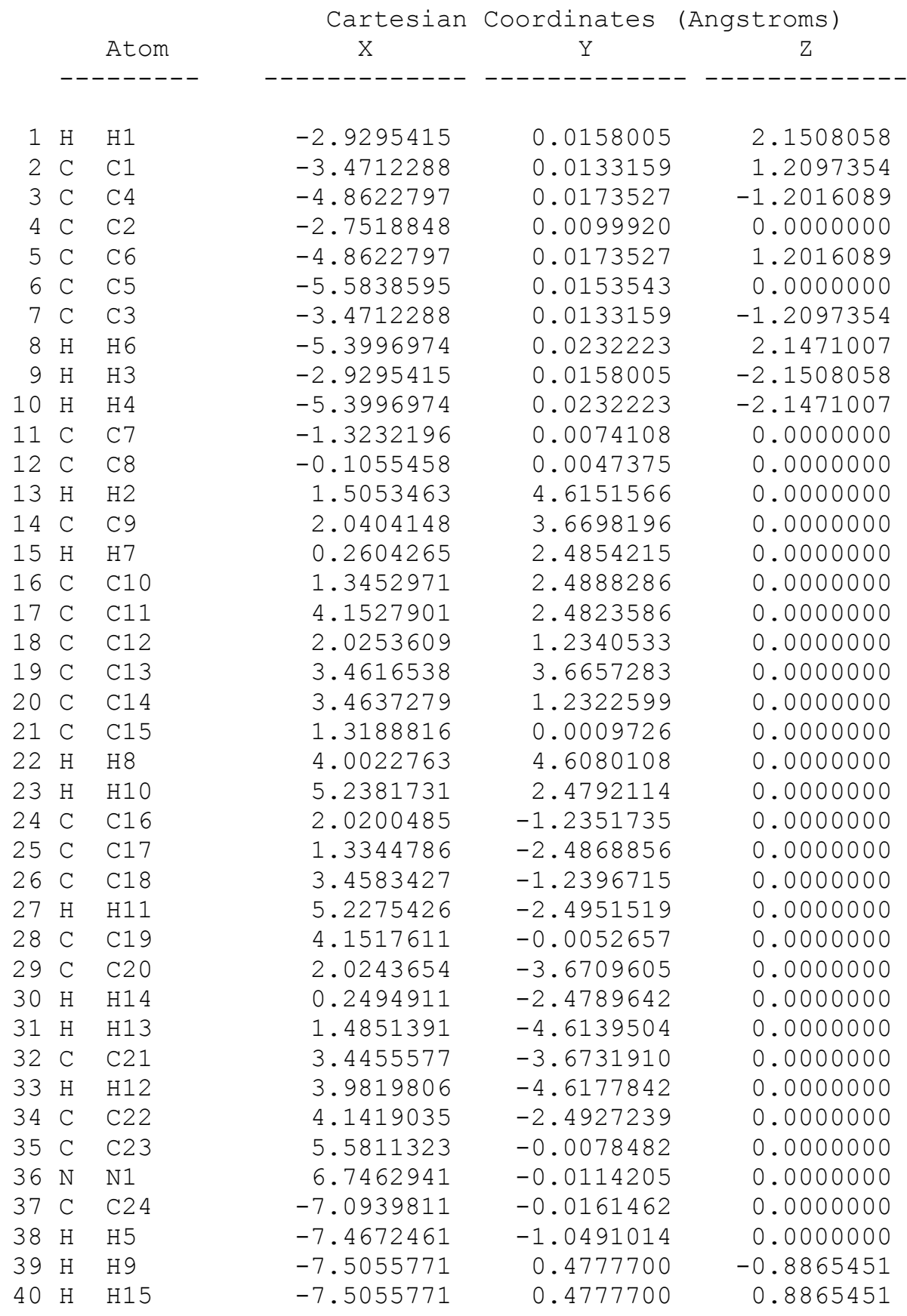


PEAN 3 (neutral, DFT/ B3LYP/6-31G*) Energy: -1053.21 au; Imaginary frequency: none

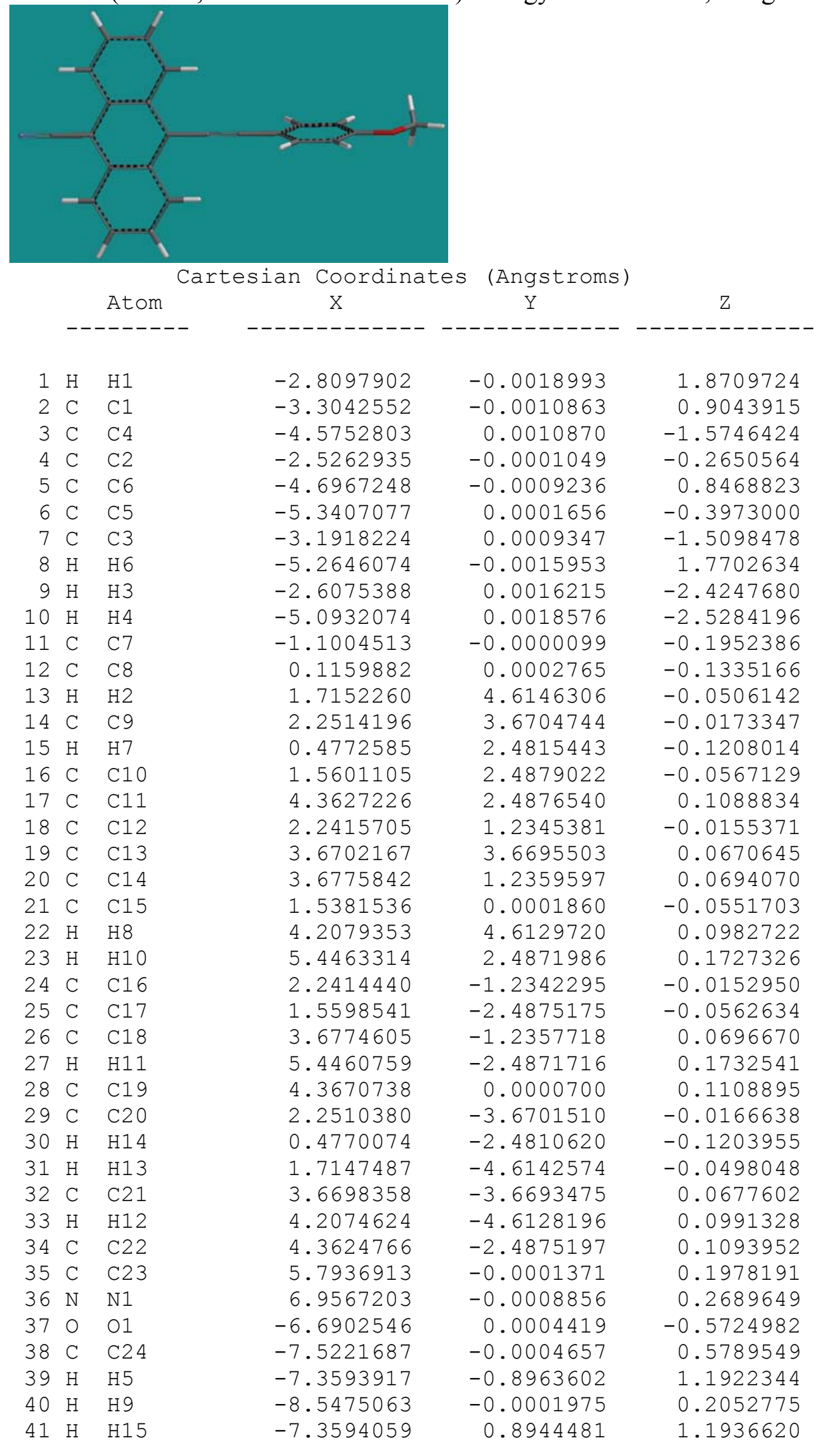


PEAN 4 (neutral, DFT/ B3LYP/6-31G*) Energy: -1072.96 au; Imaginary frequency: none

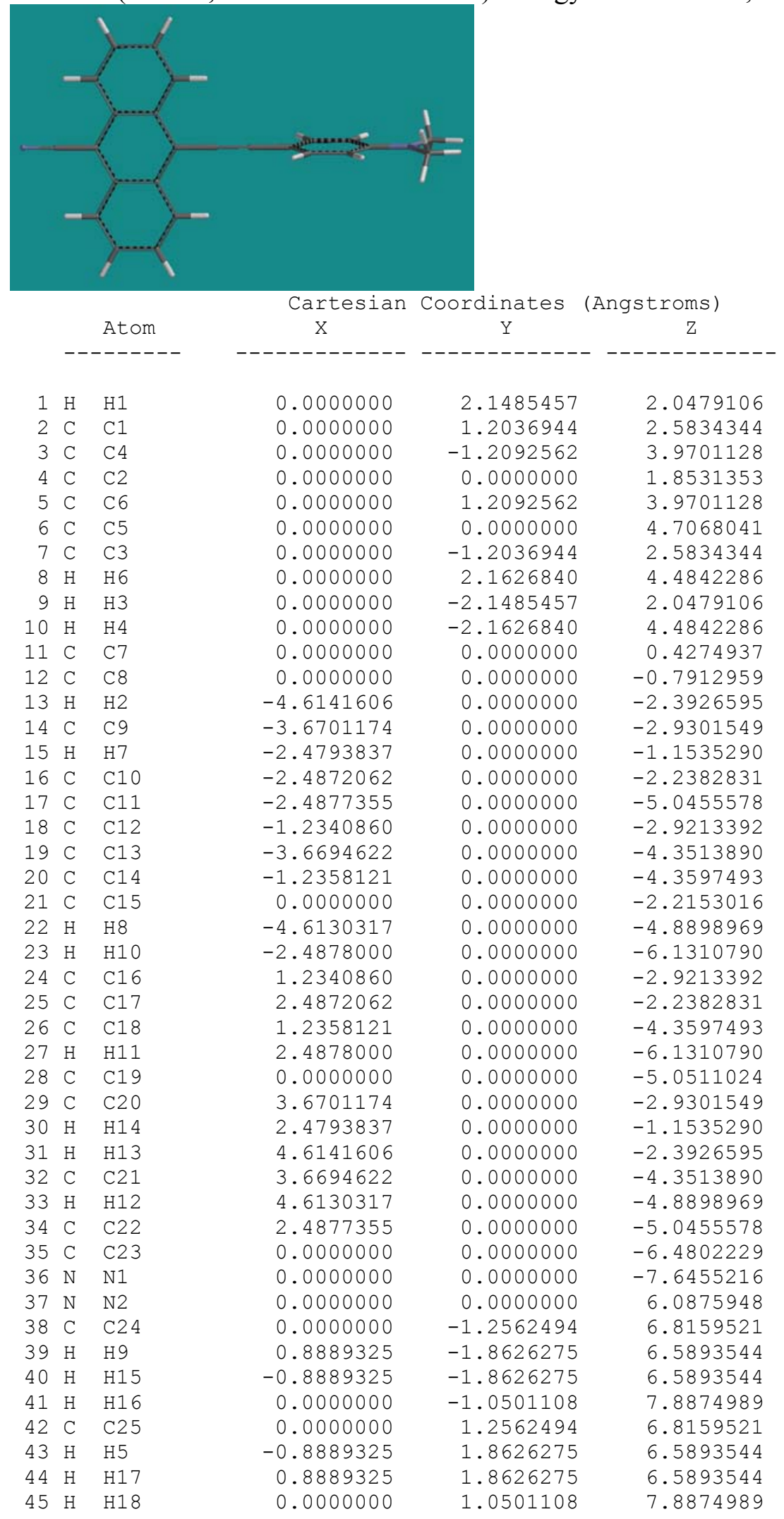


PEAN 5 (neutral, DFT/ B3LYP/6-31G*) Energy: -1151.58 au; Imaginary frequency: none

\begin{tabular}{|c|c|c|c|c|c|}
\hline & & Atom & $\begin{array}{l}\text { Cartesian } \\
\mathrm{X}\end{array}$ & $\begin{array}{c}\text { Coordinates } \\
\text { y }\end{array}$ & $\begin{array}{c}\text { (Angstroms) } \\
Z \\
-------.\end{array}$ \\
\hline 1 & $\mathrm{H}$ & $\mathrm{H} 1$ & -2.1392097 & 0.2003286 & 1.0771971 \\
\hline 2 & C & $\mathrm{C} 1$ & -1.1969440 & 0.1211371 & 1.6114320 \\
\hline 3 & C & $\mathrm{C} 4$ & 1.2001331 & -0.1130187 & 2.9983507 \\
\hline 4 & C & $\mathrm{C} 2$ & 0.0000000 & 0.0000000 & 0.8805604 \\
\hline 5 & C & C6 & -1.2001331 & 0.1130187 & 2.9983507 \\
\hline 6 & C & C5 & 0.0000000 & 0.0000000 & 3.7396017 \\
\hline 7 & $\mathrm{C}$ & C3 & 1.1969440 & -0.1211371 & 1.6114320 \\
\hline 8 & $\mathrm{H}$ & $\mathrm{H} 6$ & -2.1542224 & 0.1559788 & 3.5118688 \\
\hline 9 & $\mathrm{H}$ & $\mathrm{H} 3$ & 2.1392097 & -0.2003286 & 1.0771971 \\
\hline 10 & $\mathrm{H}$ & $\mathrm{H} 4$ & 2.1542224 & -0.1559788 & 3.5118688 \\
\hline 11 & C & C7 & 0.0000000 & 0.0000000 & -0.5450635 \\
\hline 12 & C & $\mathrm{C} 8$ & 0.0000000 & 0.0000000 & -1.7638720 \\
\hline 13 & $\mathrm{H}$ & $\mathrm{H} 2$ & -0.0471479 & -4.6139812 & -3.3653457 \\
\hline 14 & C & $\mathrm{C9}$ & -0.0365246 & -3.6699440 & -3.9027209 \\
\hline 15 & $\mathrm{H}$ & $\mathrm{H} 7$ & -0.0250271 & -2.4791082 & -2.1260617 \\
\hline 16 & C & $\mathrm{C} 10$ & -0.0242471 & -2.4870370 & -3.2108460 \\
\hline 17 & C & $\mathrm{C} 11$ & -0.0240697 & -2.4875286 & -6.0179871 \\
\hline 18 & C & $\mathrm{C} 12$ & -0.0111020 & -1.2339989 & -3.8939190 \\
\hline 19 & C & C13 & -0.0363475 & -3.6692557 & -5.3238507 \\
\hline 20 & C & C14 & -0.0114097 & -1.2356696 & -5.3321334 \\
\hline 21 & C & C15 & 0.0000000 & 0.0000000 & -3.1878766 \\
\hline 22 & $\mathrm{H}$ & H8 & -0.0464707 & -4.6127768 & -5.8623115 \\
\hline 23 & $\mathrm{H}$ & $\mathrm{H} 10$ & -0.0242971 & -2.4875667 & -7.1035314 \\
\hline 24 & C & C16 & 0.0111020 & 1.2339989 & -3.8939190 \\
\hline 25 & $\mathrm{C}$ & C17 & 0.0242471 & 2.4870370 & -3.2108460 \\
\hline 26 & $\mathrm{C}$ & $\mathrm{C} 18$ & 0.0114097 & 1.2356696 & -5.3321334 \\
\hline 27 & $\mathrm{H}$ & $\mathrm{H} 11$ & 0.0242971 & 2.4875667 & -7.1035314 \\
\hline 28 & $\mathrm{C}$ & C19 & 0.0000000 & 0.0000000 & -6.0235526 \\
\hline 29 & $\mathrm{C}$ & $\mathrm{C} 20$ & 0.0365246 & 3.6699440 & -3.9027209 \\
\hline 30 & $\mathrm{H}$ & H14 & 0.0250271 & 2.4791082 & -2.1260617 \\
\hline 31 & $\mathrm{H}$ & H13 & 0.0471479 & 4.6139812 & -3.3653457 \\
\hline 32 & $\mathrm{C}$ & C21 & 0.0363475 & 3.6692557 & -5.3238507 \\
\hline 33 & $\mathrm{H}$ & $\mathrm{H} 12$ & 0.0464707 & 4.6127768 & -5.8623115 \\
\hline 34 & $\mathrm{C}$ & C22 & 0.0240697 & 2.4875286 & -6.0179871 \\
\hline 35 & $\mathrm{C}$ & C23 & 0.0000000 & 0.0000000 & -7.4526802 \\
\hline 36 & $\mathrm{~N}$ & N1 & 0.0000000 & 0.0000000 & -8.6179882 \\
\hline 37 & $\mathrm{~N}$ & N2 & 0.0000000 & 0.0000000 & 5.1319349 \\
\hline 38 & C & $\mathrm{C} 24$ & 1.0464758 & -0.6819270 & 5.8927817 \\
\hline 39 & $\mathrm{H}$ & $\mathrm{H} 9$ & 1.6913194 & 0.0412132 & 6.4146641 \\
\hline 40 & $\mathrm{H}$ & H15 & 1.6833030 & -1.2141851 & 5.1848750 \\
\hline 41 & C & $\mathrm{C} 25$ & -1.0464758 & 0.6819270 & 5.8927817 \\
\hline 42 & $\mathrm{H}$ & H5 & -1.6913194 & -0.0412132 & 6.4146641 \\
\hline 43 & $\mathrm{H}$ & $\mathrm{H} 17$ & -1.6833030 & 1.2141851 & 5.1848750 \\
\hline 44 & C & $\mathrm{C} 26$ & 0.5002691 & -1.7047533 & 6.8996916 \\
\hline 45 & $\mathrm{H}$ & $\mathrm{H} 16$ & -0.0984552 & -1.2383758 & 7.6887042 \\
\hline 46 & $\mathrm{H}$ & H19 & 1.3352635 & -2.2233270 & 7.3845246 \\
\hline 47 & $\mathrm{H}$ & $\mathrm{H} 2 \mathrm{O}$ & -0.1214266 & -2.4514696 & 6.3940858 \\
\hline 48 & $\mathrm{C}$ & $\mathrm{C} 27$ & -0.5002691 & 1.7047533 & 6.8996916 \\
\hline 49 & $\mathrm{H}$ & $\mathrm{H} 18$ & 0.1214266 & 2.4514696 & 6.3940858 \\
\hline 50 & $\mathrm{H}$ & H21 & 0.0984552 & 1.2383758 & 7.6887042 \\
\hline 51 & $\mathrm{H}$ & H22 & -1.3352635 & 2.2233270 & 7.3845246 \\
\hline
\end{tabular}


PEAN 6 (neutral, DFT/ B3LYP/6-31G*) Energy: -1189.69 au; Imaginary frequency: none

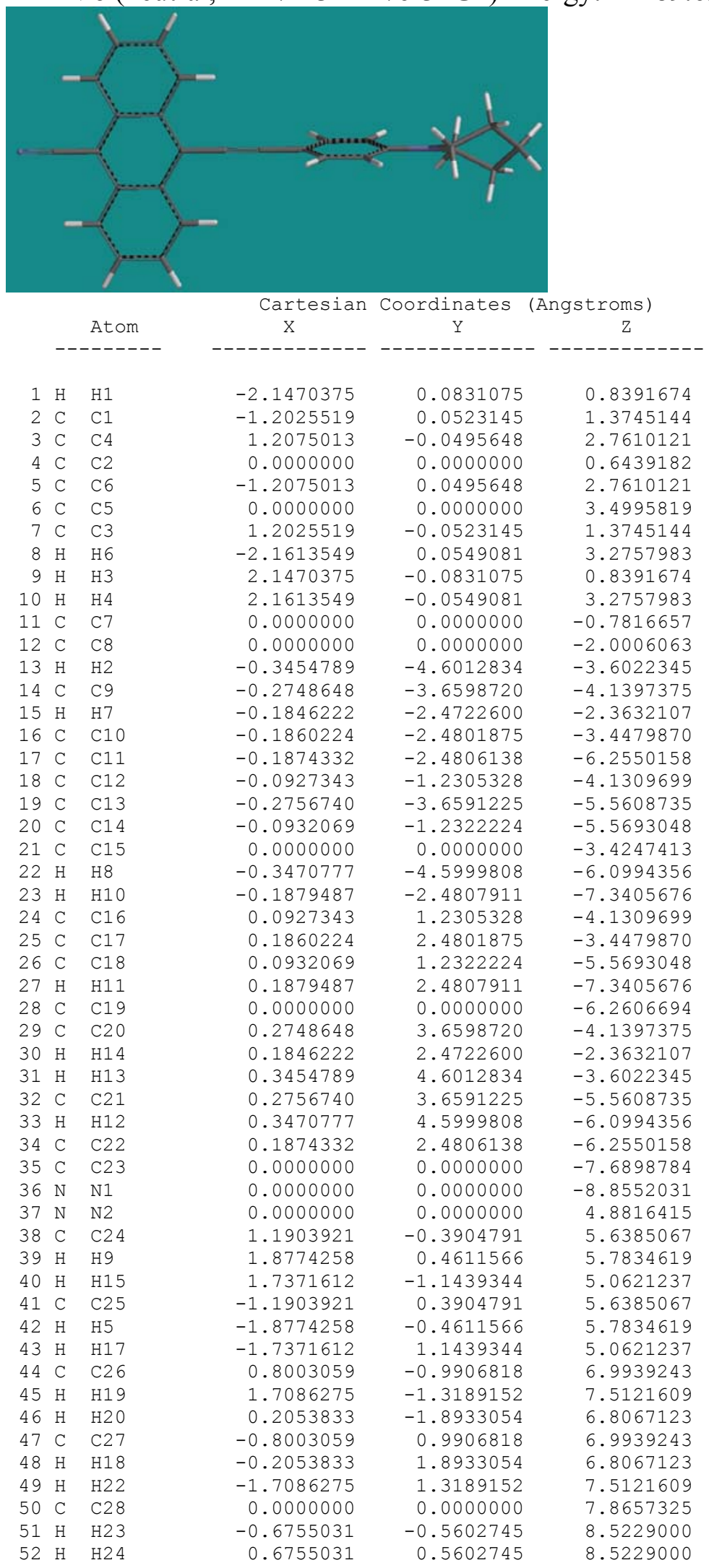


Radical anion of 1: (DFT - B3LYP/6-31G*) Energy: -939.039 au; Imaginary frequencies: none

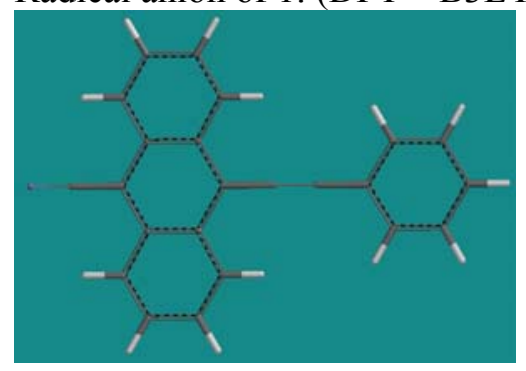

Molecular charge: -1

Spin multiplicity: 2

\begin{tabular}{|c|c|c|c|c|}
\hline & Atom & $\begin{array}{l}\text { Cartesian } \\
\quad \mathrm{x}\end{array}$ & $\begin{array}{c}\text { Coordinates } \\
y\end{array}$ & $\begin{array}{c}\text { (Angstroms) } \\
\mathrm{Z}\end{array}$ \\
\hline $1 \mathrm{H}$ & $\mathrm{H} \quad \mathrm{H} 1$ & -4.6363704 & 0.0000000 & -3.3803225 \\
\hline $2 c$ & $\mathrm{C} \quad \mathrm{C} 1$ & -3.6981524 & 0.0000000 & -2.8291372 \\
\hline $3 \mathrm{H}$ & H H6 & -2.4919841 & 0.0000000 & -4.6029465 \\
\hline $4 c$ & C $\quad$ C6 & -2.4963346 & 0.0000000 & -3.5162549 \\
\hline 50 & $\mathrm{C} \quad \mathrm{C} 3$ & -2.4995377 & 0.0000000 & -0.7341298 \\
\hline 60 & C C5 & -1.2510960 & 0.0000000 & -2.8384436 \\
\hline 70 & C $\quad$ C2 & -3.7010848 & 0.0000000 & -1.4233774 \\
\hline 80 & C $\quad$ C4 & -1.2529242 & 0.0000000 & -1.4038134 \\
\hline 90 & C C10 & 0.0000000 & 0.0000000 & -3.5441293 \\
\hline $10 \mathrm{~F}$ & $\mathrm{H} \quad \mathrm{H} 2$ & -4.6411159 & 0.0000000 & -0.8755282 \\
\hline $11 \mathrm{H}$ & H H3 & -2.4947486 & 0.0000000 & 0.3519783 \\
\hline 120 & C C9 & 1.2510960 & 0.0000000 & -2.8384436 \\
\hline $13 c$ & C $\quad$ C14 & 2.4963346 & 0.0000000 & -3.5162549 \\
\hline 140 & C C 8 & 1.2529242 & 0.0000000 & -1.4038134 \\
\hline 15 & $\mathrm{H} \quad \mathrm{H} 11$ & 2.4947486 & 0.0000000 & 0.3519783 \\
\hline 160 & $\mathrm{C} \quad \mathrm{C} 7$ & 0.0000000 & 0.0000000 & -0.6809629 \\
\hline 17 & C $\mathrm{C} 13$ & 3.6981524 & 0.0000000 & -2.8291372 \\
\hline $18 \mathrm{H}$ & $\mathrm{H} \quad \mathrm{H} 14$ & 2.4919841 & 0.0000000 & -4.6029465 \\
\hline $19 \mathrm{H}$ & $\mathrm{H} \quad \mathrm{H} 13$ & 4.6363704 & 0.0000000 & -3.3803225 \\
\hline 20 & C $\quad$ C12 & 3.7010848 & 0.0000000 & -1.4233774 \\
\hline $21 \mathrm{H}$ & $\mathrm{H} \quad \mathrm{H} 12$ & 4.6411159 & 0.0000000 & -0.8755282 \\
\hline 22 & C $\quad$ C11 & 2.4995377 & 0.0000000 & -0.7341298 \\
\hline $23 c$ & C $\quad$ C15 & 0.0000000 & 0.0000000 & 0.7169722 \\
\hline $24 c$ & C $\mathrm{C} 16$ & 0.0000000 & 0.0000000 & 1.9470639 \\
\hline 25 & C $\quad$ C17 & 0.0000000 & 0.0000000 & 3.3567914 \\
\hline $26 c$ & C $\quad$ C18 & 0.0000000 & 0.0000000 & 6.1972070 \\
\hline 27 & C $\mathrm{C} 19$ & -1.2122767 & 0.0000000 & 4.0959384 \\
\hline 28 & C $\quad$ C20 & 1.2122767 & 0.0000000 & 4.0959384 \\
\hline 290 & C $\quad$ C21 & 1.2053447 & 0.0000000 & 5.4854007 \\
\hline $30 c$ & C $\quad$ C22 & -1.2053447 & 0.0000000 & 5.4854007 \\
\hline 31 & H $\quad$ H4 & -2.1535941 & 0.0000000 & 3.5537682 \\
\hline 32 & H H5 & 2.1535941 & 0.0000000 & 3.5537682 \\
\hline $33 \mathrm{f}$ & $\mathrm{H} \quad \mathrm{H} 7$ & 2.1520334 & 0.0000000 & 6.0223934 \\
\hline $34 \mathrm{H}$ & H H8 & -2.1520334 & 0.0000000 & 6.0223934 \\
\hline $35 \mathrm{H}$ & H H9 & 0.0000000 & 0.0000000 & 7.2843824 \\
\hline 36 & C $\quad$ C23 & 0.0000000 & 0.0000000 & -4.9579313 \\
\hline 37 & $\mathrm{~N} \quad \mathrm{~N} 1$ & 0.0000000 & 0.0000000 & -6.1304445 \\
\hline
\end{tabular}


Radical cation of 1: (DFT - B3LYP/6-31G*) Energy: -939.741 au; Imaginary frequencies: none

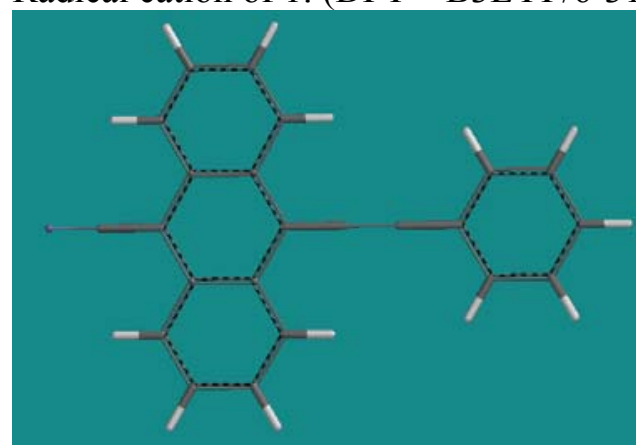

Molecular charge: 1

Spin multiplicity: 2

\begin{tabular}{|c|c|c|c|c|}
\hline & Atom & $\begin{array}{l}\text { Cartesian } \\
\quad \mathrm{X}\end{array}$ & $\begin{array}{c}\text { Coordinates } \\
Y\end{array}$ & $\begin{array}{c}\text { Angstroms ) } \\
\text { Z }\end{array}$ \\
\hline & --------- & ------------- & ---------- & - \\
\hline 1 & $\mathrm{H} 1$ & -4.6158088 & 0.0000000 & -3.3744012 \\
\hline 2 & $\mathrm{C} 1$ & -3.6783865 & 0.0000000 & -2.8276994 \\
\hline 3 & $\mathrm{H} 6$ & -2.4755371 & 0.0000000 & -4.6026029 \\
\hline 4 & C6 & -2.4793930 & 0.0000000 & -3.5173688 \\
\hline 5 & $\mathrm{C} 3$ & -2.4902548 & 0.0000000 & -0.7153184 \\
\hline 6 & $\mathrm{C} 5$ & -1.2447661 & 0.0000000 & -2.8197193 \\
\hline 7 & $\mathrm{C} 2$ & -3.6835983 & 0.0000000 & -1.4211268 \\
\hline 8 & $\mathrm{C} 4$ & -1.2494123 & 0.0000000 & -1.3860660 \\
\hline 9 & $\mathrm{C} 10$ & 0.0000000 & 0.0000000 & -3.5111450 \\
\hline 10 & $\mathrm{H} 2$ & -4.6267794 & 0.0000000 & -0.8839575 \\
\hline 11 & $\mathrm{H} 3$ & -2.5011512 & 0.0000000 & 0.3689341 \\
\hline 12 & $\mathrm{C} 9$ & 1.2447661 & 0.0000000 & -2.8197193 \\
\hline 13 & $\mathrm{C} 14$ & 2.4793930 & 0.0000000 & -3.5173688 \\
\hline 14 & $\mathrm{C} 8$ & 1.2494123 & 0.0000000 & -1.3860660 \\
\hline 15 & $\mathrm{H} 11$ & 2.5011512 & 0.0000000 & 0.3689341 \\
\hline 16 & C7 & 0.0000000 & 0.0000000 & -0.6667975 \\
\hline 17 & C13 & 3.6783865 & 0.0000000 & -2.8276994 \\
\hline 18 & $\mathrm{H} 14$ & 2.4755371 & 0.0000000 & -4.6026029 \\
\hline 19 & $\mathrm{H} 13$ & 4.6158088 & 0.0000000 & -3.3744012 \\
\hline 20 & $\mathrm{C} 12$ & 3.6835983 & 0.0000000 & -1.4211268 \\
\hline 21 & $\mathrm{H} 12$ & 4.6267794 & 0.0000000 & -0.8839575 \\
\hline 22 & C11 & 2.4902548 & 0.0000000 & -0.7153184 \\
\hline 23 & C15 & 0.0000000 & 0.0000000 & 0.7257617 \\
\hline 24 & C16 & 0.0000000 & 0.0000000 & 1.9544173 \\
\hline 25 & C17 & 0.0000000 & 0.0000000 & 3.3604137 \\
\hline 26 & C18 & 0.0000000 & 0.0000000 & 6.1544661 \\
\hline 27 & C19 & -1.2250932 & 0.0000000 & 4.0742860 \\
\hline 28 & $\mathrm{C} 20$ & 1.2250932 & 0.0000000 & 4.0742860 \\
\hline 29 & $\mathrm{C} 21$ & 1.2171173 & 0.0000000 & 5.4606941 \\
\hline 30 & $\mathrm{C} 22$ & -1.2171173 & 0.0000000 & 5.4606941 \\
\hline 31 & $\mathrm{H} 4$ & -2.1621182 & 0.0000000 & 3.5270417 \\
\hline 32 & H5 & 2.1621182 & 0.0000000 & 3.5270417 \\
\hline 33 & $\mathrm{H} 7$ & 2.1543537 & 0.0000000 & 6.0080412 \\
\hline 34 & H8 & -2.1543537 & 0.0000000 & 6.0080412 \\
\hline 35 & H 9 & 0.0000000 & 0.0000000 & 7.2405547 \\
\hline 36 & $\mathrm{C} 23$ & 0.0000000 & 0.0000000 & -4.9372128 \\
\hline 37 & $\mathrm{~N} 1$ & 0.0000000 & 0.0000000 & -6.1019317 \\
\hline
\end{tabular}


Radical anion of 6: (DFT - B3LYP/6-31G*) Energy: -1189.753 au; Imaginary frequencies: none

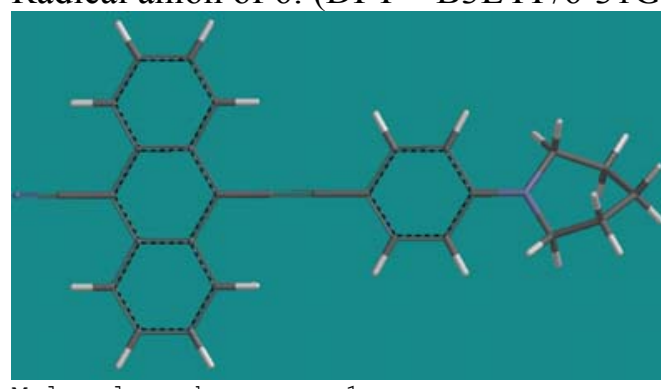

Molecular charge: -1

Spin multiplicity: 2

\begin{tabular}{|c|c|c|c|c|c|}
\hline & & & Cartesian & Coordinates & gstroms) \\
\hline & & Atom & X & $\mathrm{Y}$ & Z \\
\hline & & ------- & -------- & $5-2-5=$ & \\
\hline 1 & $\mathrm{H}$ & $\mathrm{H} 1$ & -0.8610524 & -2.1560491 & 0.0761708 \\
\hline 2 & $\mathrm{C}$ & $\mathrm{C} 1$ & -1.3852372 & -1.2047598 & 0.0550142 \\
\hline 3 & $\mathrm{C}$ & $\mathrm{C} 4$ & -2.7533915 & 1.2127715 & 0.0200739 \\
\hline 4 & $\mathrm{C}$ & $\mathrm{C} 2$ & -0.6270837 & -0.0094703 & 0.0221764 \\
\hline 5 & $\mathrm{C}$ & C6 & -2.7733462 & -1.1941659 & 0.0796168 \\
\hline 6 & $\mathrm{C}$ & $\mathrm{C} 5$ & -3.5003626 & 0.0158567 & 0.0594549 \\
\hline 7 & $\mathrm{C}$ & $\mathrm{C} 3$ & -1.3647794 & 1.1985417 & 0.0081731 \\
\hline 8 & $\mathrm{H}$ & H6 & -3.2993809 & -2.1421529 & 0.1431606 \\
\hline 9 & $\mathrm{H}$ & H3 & -0.8247417 & 2.1405614 & -0.0265343 \\
\hline 10 & $\mathrm{H}$ & $\mathrm{H} 4$ & -3.2624165 & 2.1705873 & -0.0275373 \\
\hline 11 & $\mathrm{C}$ & C7 & 0.7855717 & -0.0212110 & 0.0051913 \\
\hline 12 & $\mathrm{C}$ & $\mathrm{C} 8$ & 2.0145768 & -0.0292449 & -0.0071416 \\
\hline 13 & $\mathrm{H}$ & $\mathrm{H} 2$ & 3.6272678 & 4.6050733 & -0.0779699 \\
\hline 14 & $\mathrm{C}$ & C9 & 4.1718048 & 3.6629579 & -0.0723634 \\
\hline 15 & $\mathrm{H}$ & $\mathrm{H} 7$ & 2.3918225 & 2.4631708 & -0.0404095 \\
\hline 16 & $\mathrm{C}$ & $\mathrm{C} 10$ & 3.4779775 & 2.4634514 & -0.0512874 \\
\hline 17 & $\mathrm{C}$ & C11 & 6.2592389 & 2.4488930 & -0.0794950 \\
\hline 18 & $\mathrm{C}$ & C12 & 4.1425691 & 1.2139094 & -0.0433488 \\
\hline 19 & $\mathrm{C}$ & C13 & 5.5766246 & 3.6544086 & -0.0865739 \\
\hline 20 & $\mathrm{C}$ & C14 & 5.5776371 & 1.2065485 & -0.0580541 \\
\hline 21 & $\mathrm{C}$ & C15 & 3.4155388 & -0.0358609 & -0.0214869 \\
\hline 22 & $\mathrm{H}$ & $\mathrm{H} 8$ & 6.1318578 & 4.5902677 & -0.1033554 \\
\hline 23 & $\mathrm{H}$ & $\mathrm{H} 10$ & 7.3459235 & 2.4406946 & -0.0906456 \\
\hline 24 & $\mathrm{C}$ & C16 & 4.1320362 & -1.2916819 & -0.0131304 \\
\hline 25 & $\mathrm{C}$ & C17 & 3.4568347 & -2.5353289 & 0.0092566 \\
\hline 26 & $\mathrm{C}$ & $\mathrm{C} 18$ & 5.5671461 & -1.2965904 & -0.0277920 \\
\hline 27 & $\mathrm{H}$ & H11 & 7.3249329 & -2.5462423 & -0.0300711 \\
\hline 28 & $\mathrm{C}$ & C19 & 6.2785385 & -0.0480563 & -0.0505418 \\
\hline 29 & $\mathrm{C}$ & $\mathrm{C} 20$ & 4.1405515 & -3.7407651 & 0.0177242 \\
\hline 30 & $\mathrm{H}$ & $\mathrm{H} 14$ & 2.3706979 & -2.5253580 & 0.0201123 \\
\hline 31 & $\mathrm{H}$ & H13 & 3.5880995 & -4.6780896 & 0.0356423 \\
\hline 32 & $\mathrm{C}$ & $\mathrm{C} 21$ & 5.5454425 & -3.7443468 & 0.0032642 \\
\hline 33 & $\mathrm{H}$ & $\mathrm{H} 12$ & 6.0928285 & -4.6849597 & 0.0095499 \\
\hline 34 & $\mathrm{C}$ & $\mathrm{C} 22$ & 6.2382080 & -2.5448173 & -0.0189287 \\
\hline 35 & $\mathrm{C}$ & $\mathrm{C} 23$ & 7.6916211 & -0.0543767 & -0.0653114 \\
\hline 36 & $\mathrm{~N}$ & N1 & 8.8643933 & -0.0607661 & -0.0777073 \\
\hline 37 & $\mathrm{~N}$ & $\mathrm{~N} 2$ & -4.9004900 & 0.0249317 & 0.0841351 \\
\hline 38 & $\mathrm{C}$ & $\mathrm{C} 24$ & -5.6320458 & 1.2323035 & 0.4344857 \\
\hline 39 & $\mathrm{H}$ & H9 & -5.7538935 & 1.9191553 & -0.4255829 \\
\hline 40 & $\mathrm{H}$ & H1 5 & -5.0551217 & 1.7812520 & 1.1877547 \\
\hline 41 & $\mathrm{C}$ & $\mathrm{C} 25$ & -5.6623585 & -1.1420802 & -0.3400671 \\
\hline 42 & $\mathrm{H}$ & H5 & -5.8458074 & -1.8467243 & 0.4930379 \\
\hline 43 & $\mathrm{H}$ & H1 7 & -5.0747204 & -1.6906316 & -1.0853934 \\
\hline 44 & $\mathrm{C}$ & $\mathrm{C} 26$ & -7.0082329 & 0.8833724 & 1.0138052 \\
\hline 45 & $\mathrm{H}$ & H19 & -7.5185028 & 1.8058758 & 1.3169042 \\
\hline 46 & $\mathrm{H}$ & H2O & -6.8516096 & 0.2967034 & 1.9278135 \\
\hline 47 & $\mathrm{C}$ & $\mathrm{C} 27$ & -6.9992768 & -0.7246286 & -0.9655024 \\
\hline 48 & $\mathrm{H}$ & H1 8 & -6.7824569 & -0.1213761 & -1.8560676 \\
\hline 49 & $\mathrm{H}$ & H22 & -7.5320732 & -1.6178970 & -1.3143146 \\
\hline 50 & $\mathrm{C}$ & $\mathrm{C} 28$ & -7.8750219 & 0.0835426 & 0.0157197 \\
\hline 51 & $\mathrm{H}$ & H23 & -8.5417332 & -0.5886077 & 0.5706602 \\
\hline 52 & $\mathrm{H}$ & H24 & -8.5246048 & 0.7614086 & -0.5522839 \\
\hline
\end{tabular}

Point Group $=\mathrm{C} 1$ Order $=1$ Nsymop $=1$ 
Radican cation of 6: (DFT - B3LYP/6-31G*) Energy: -1189.471 au; Imaginary frequencies: none

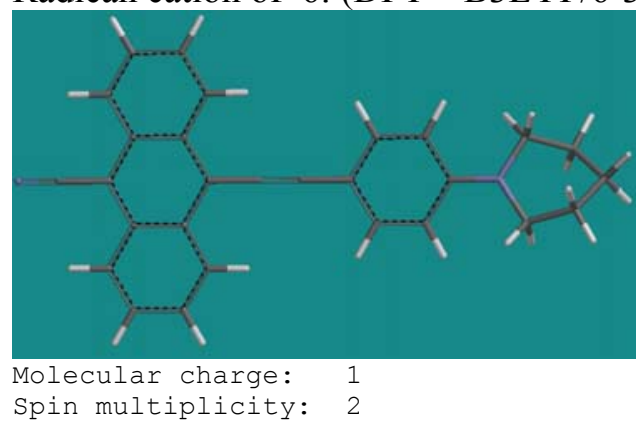

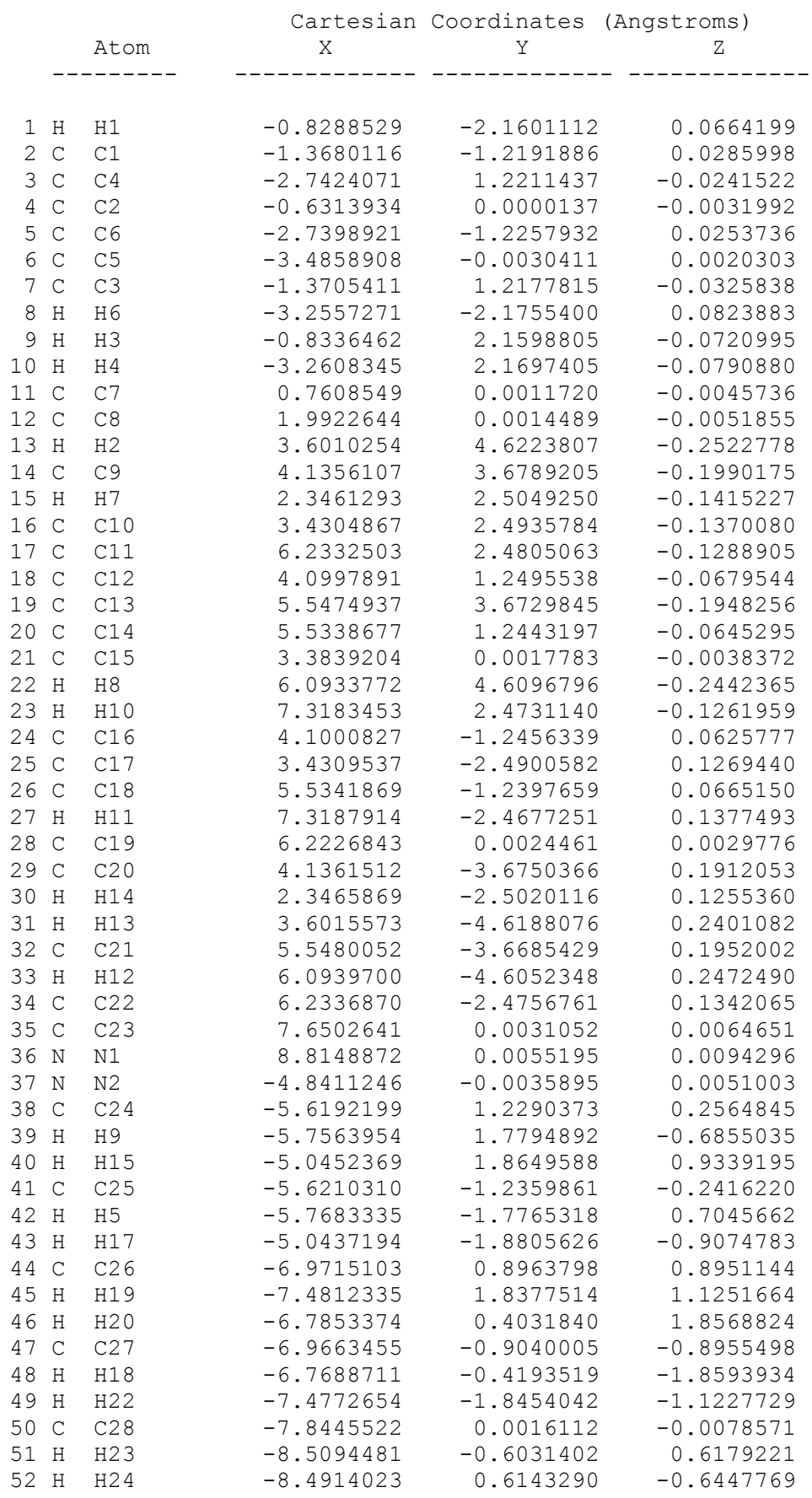

Point Group $=\mathrm{C} 1$ Order $=1$ Nsymop $=1$ 


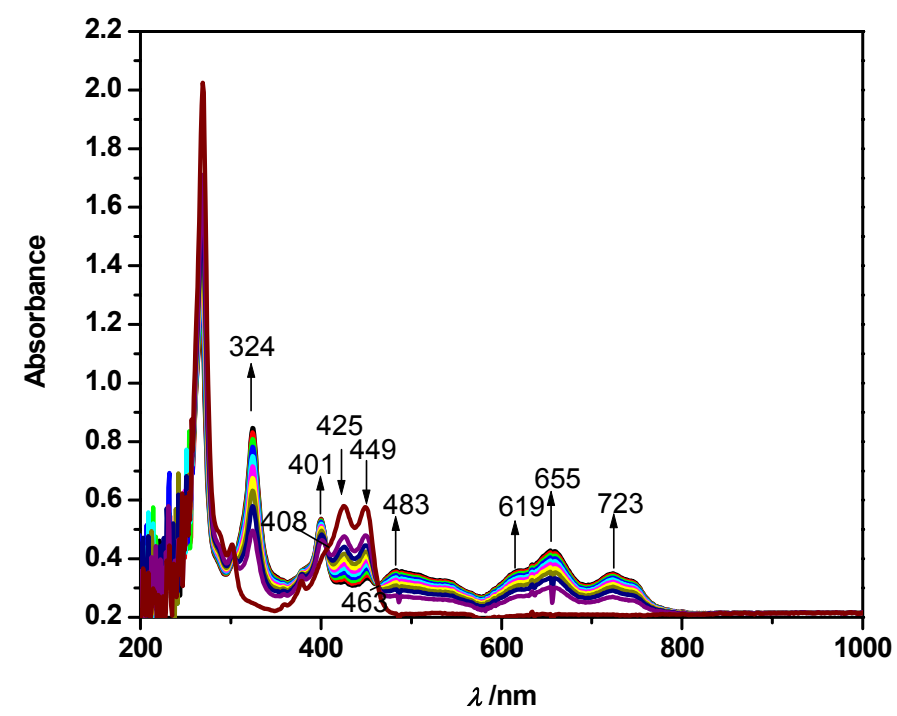

Figure S6-1. UV-Vis Spectra of radical anion of $\mathbf{1}\left(0.5 \mathrm{mM}, \mathrm{CH}_{3} \mathrm{CN}+0.5 \mathrm{M}\right.$ TBAP $)$.

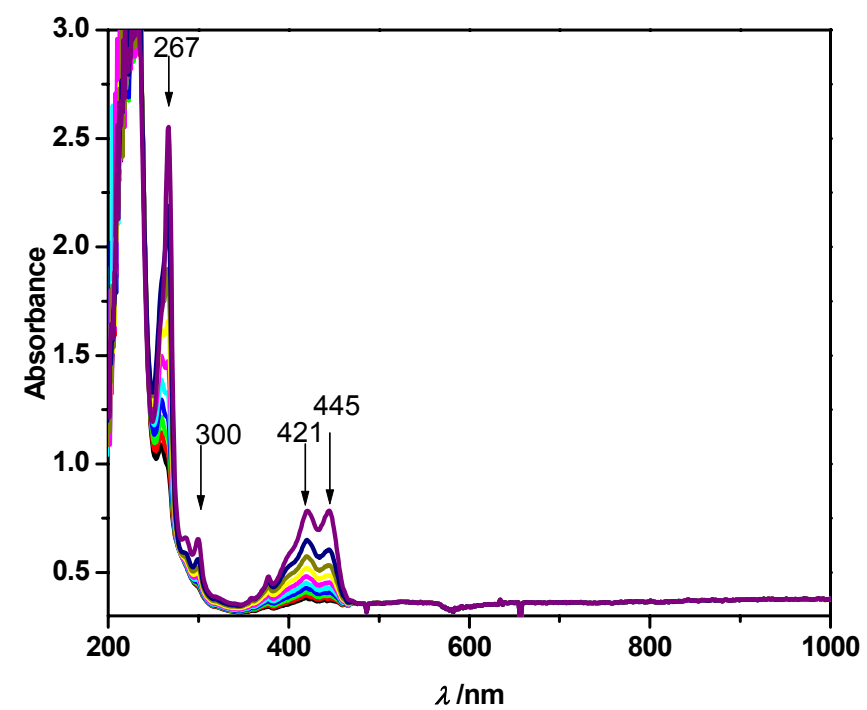

Figure S6-2. UV-Vis Spectra of radical cation of $\mathbf{1}\left(0.5 \mathrm{mM}, \mathrm{CH}_{3} \mathrm{CN}+0.5 \mathrm{M}\right.$ TBAP $)$. 


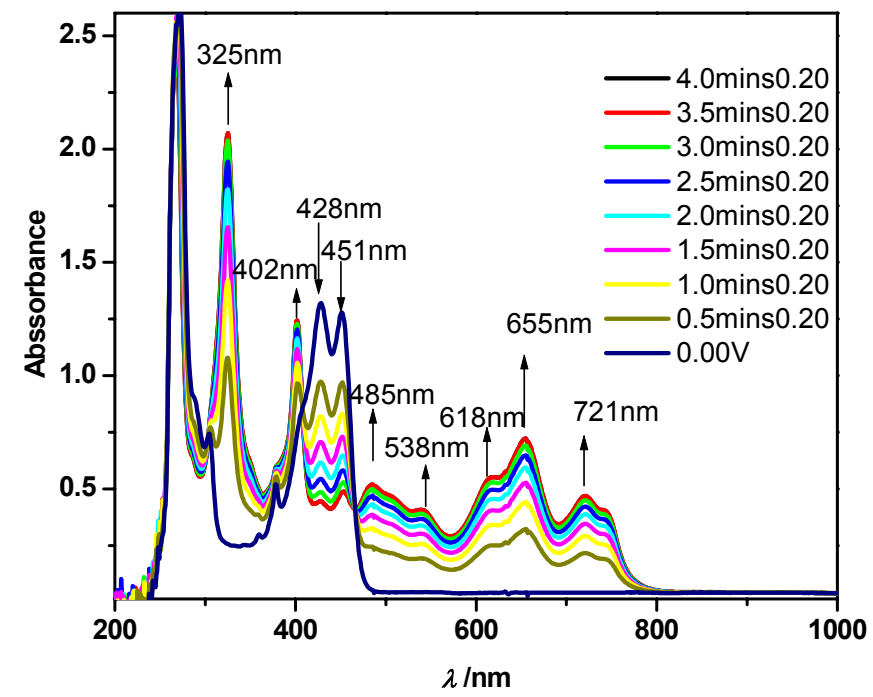

Figure S6-3. UV-Vis Spectra of radical anion of 2 ( $0.5 \mathrm{mM}, \mathrm{DMF}+0.1 \mathrm{M}$ TBAP).

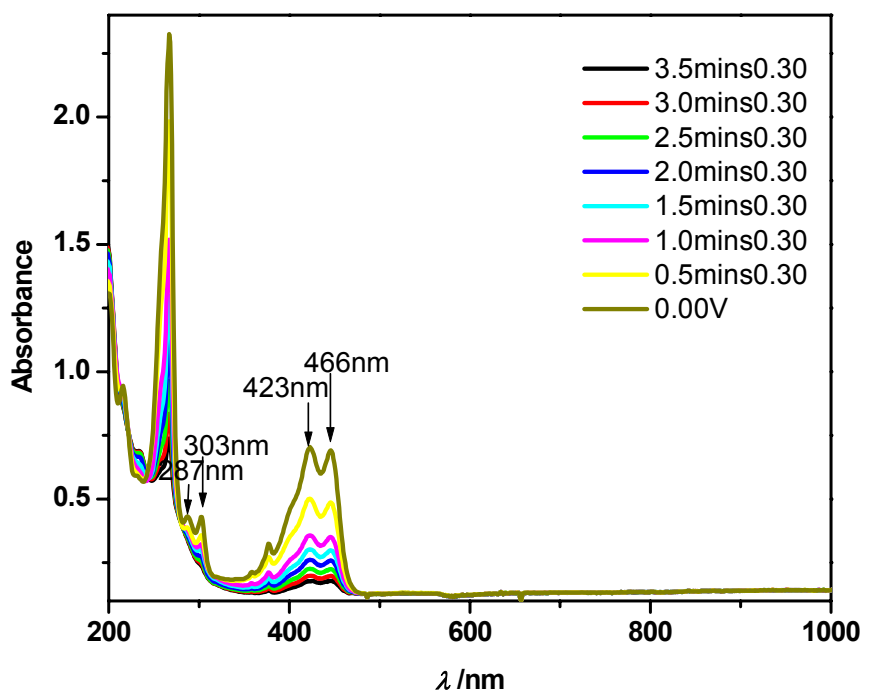

Figure S6-4. UV-Vis Spectra of radical cation of $2\left(0.5 \mathrm{mM}, \mathrm{CH}_{3} \mathrm{CN}+0.1 \mathrm{M}\right.$ TBAP). 


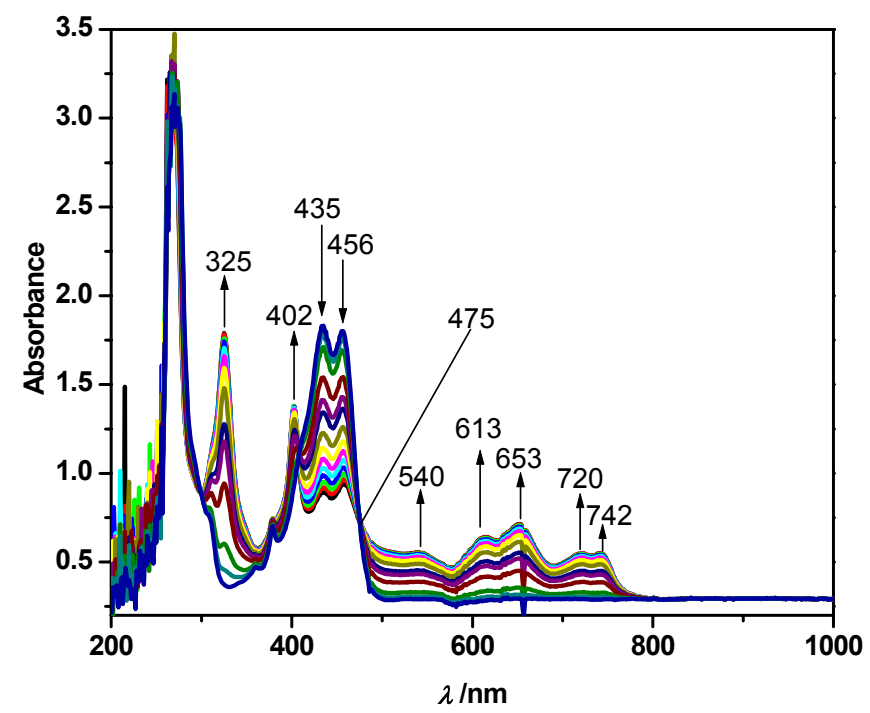

Figure S6-5. UV-Vis Spectra of radical anion of 3 (0.5 mM, DMF + 0.5M TBAP).

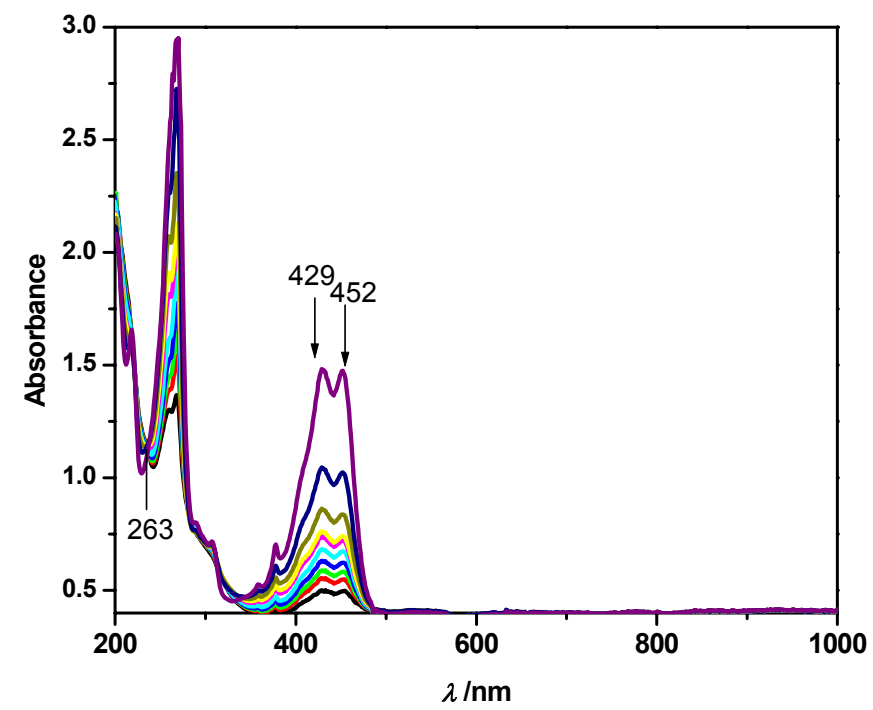

Figure S6-6. UV-Vis Spectra of radical cation of 3 (0.5 mM, DMF + 0.5M TBAP). 


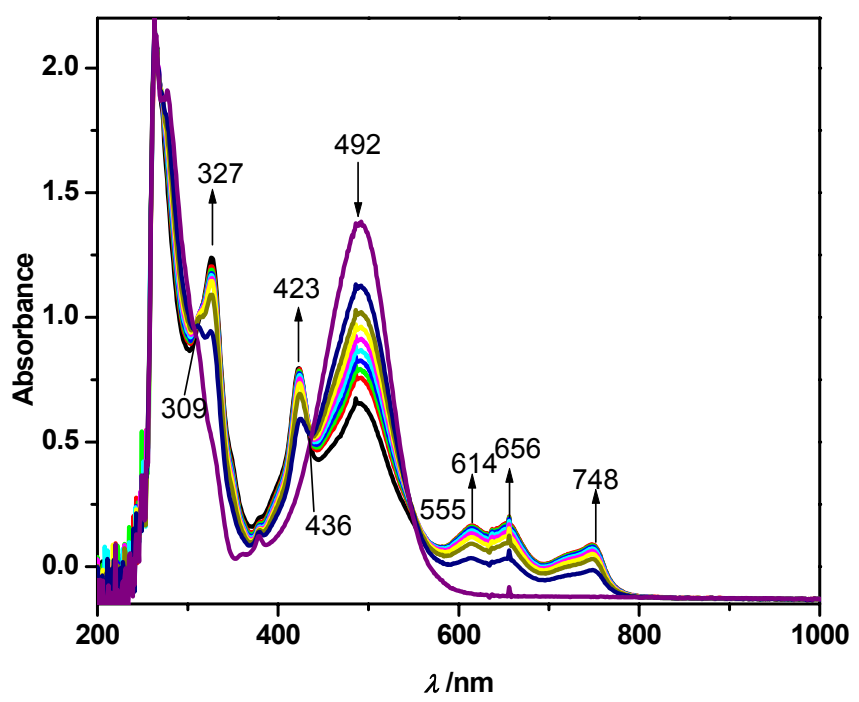

Figure S6-7. UV-Vis Spectra of radical anion of 4 (0.5 mM, DMF + 0.5M TBAP).

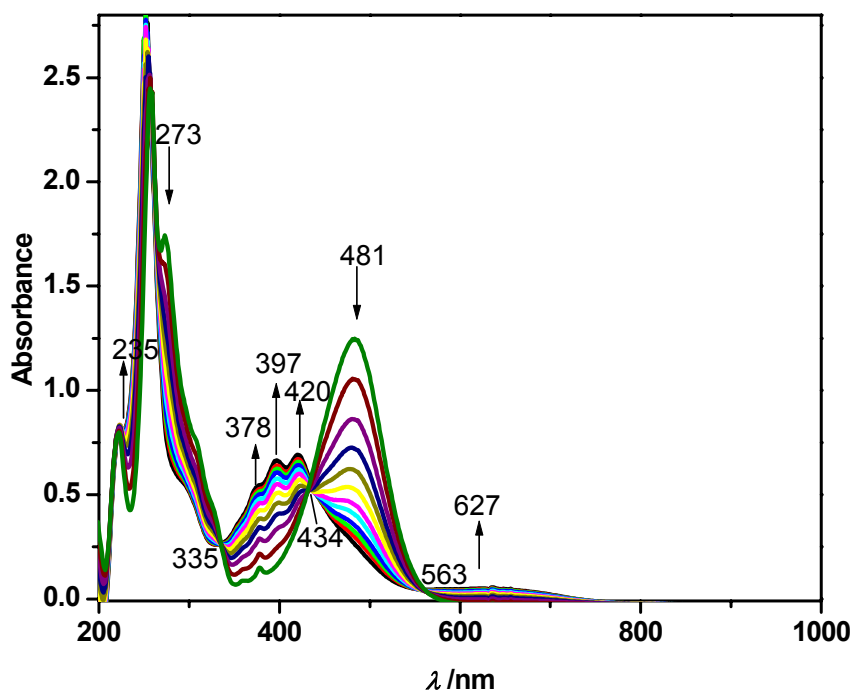

Figure S6-8. UV-Vis Spectra of radical cation of 4 (0.5 mM, DMF + 0.5M TBAP). 


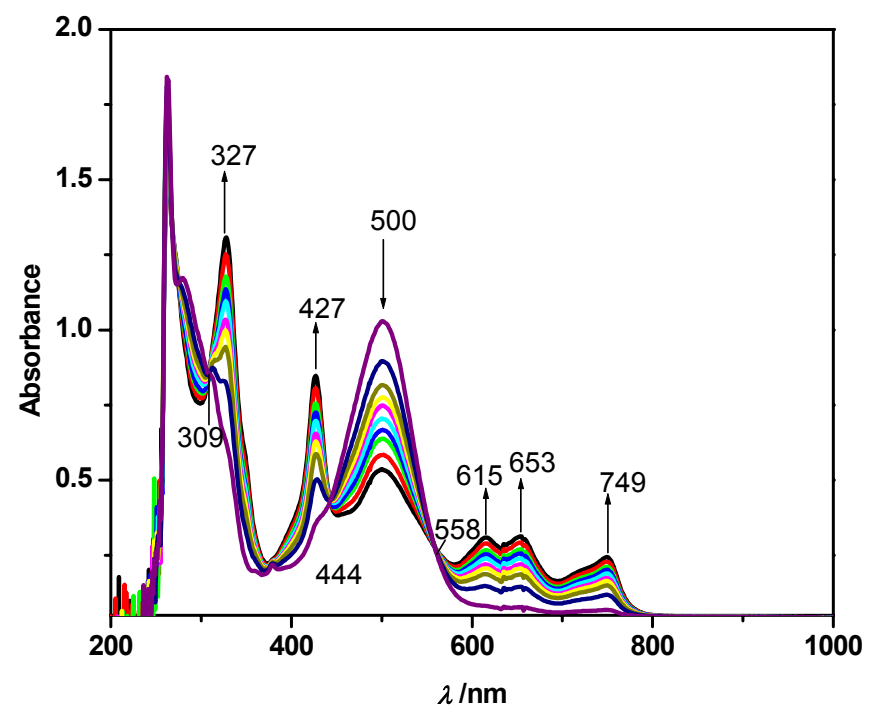

Figure S6-9. UV-Vis Spectra of radical anion of 5 (0.5 mM, DMF + 0.5M TBAP).

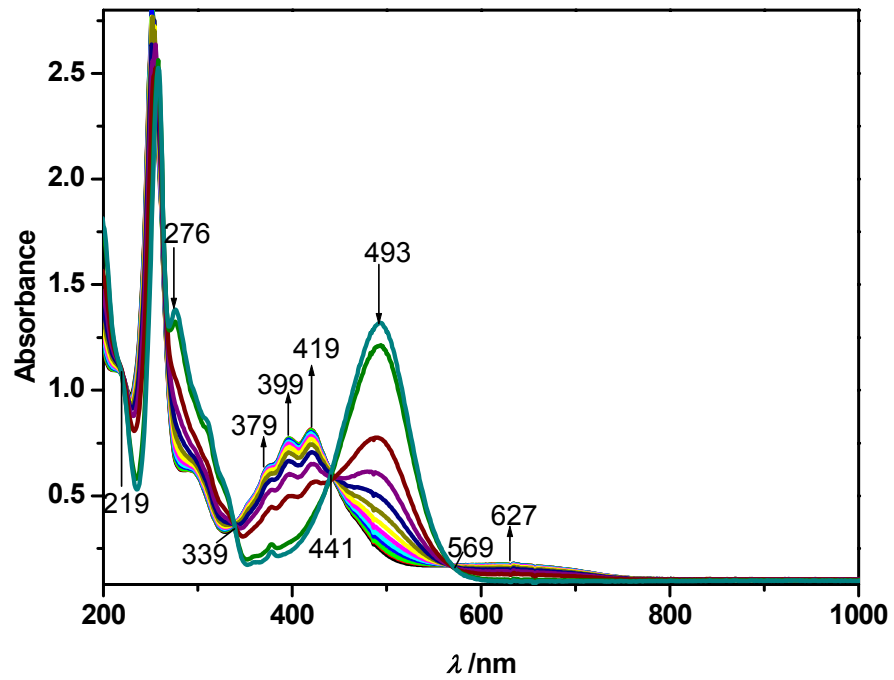

Figure S6-10. UV-Vis Spectra of radical cation of $5(0.5 \mathrm{mM}, \mathrm{MeCN}+0.5 \mathrm{M}$ TBAP). 


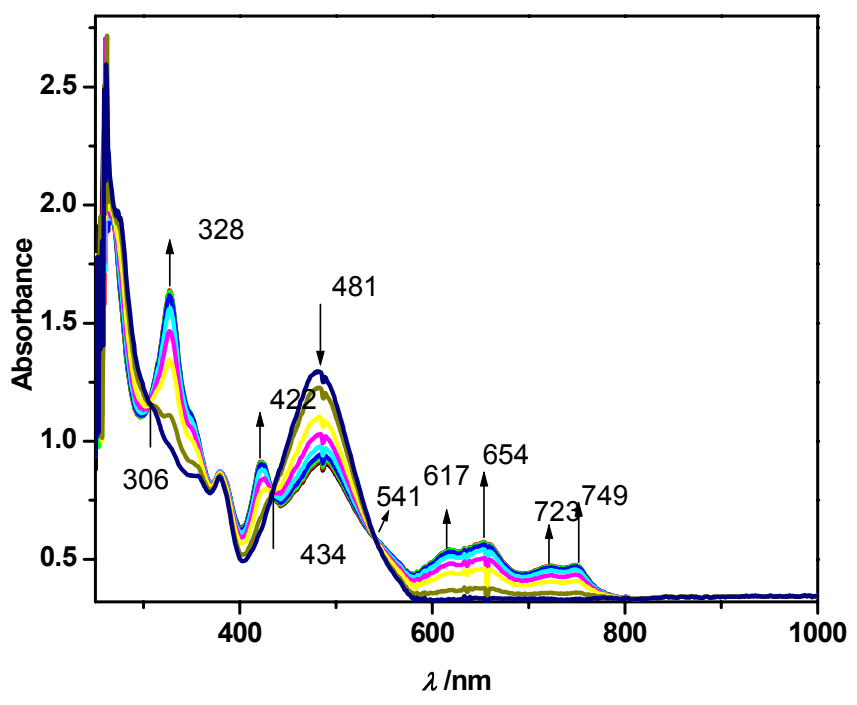

Figure S6-11. UV-Vis Spectra of radical anion of $6(0.5 \mathrm{mM}, \mathrm{DMF}+0.5 \mathrm{M}$ TBAP $)$.

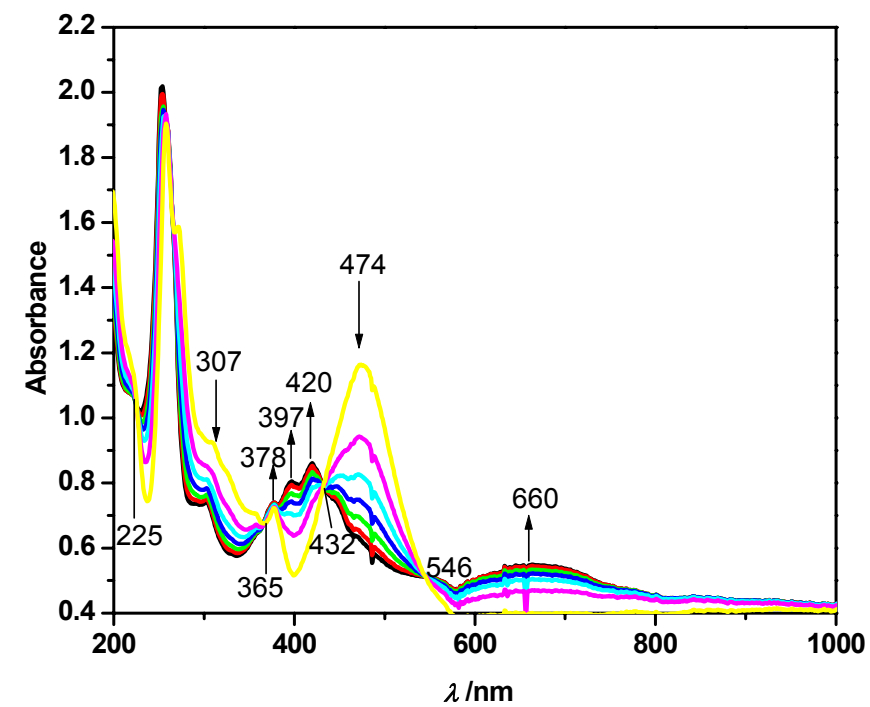

Figure S6-12. UV-Vis Spectra of radical cation of $6(0.5 \mathrm{mM}, \mathrm{MeCN}+0.5 \mathrm{M}$ TBAP). 